\title{
Analysis of the genomic basis of functional diversity in dinoflagellates using a transcriptome-based sequence similarity network
}

\author{
Meng Arnaud ${ }^{1,}{ }^{*}$, Corre Erwan ${ }^{2}$, Probert lan ${ }^{3}$, Gutierrez-Rodriguez Andres ${ }^{4}$, Siano Raffaele ${ }^{5}$, \\ Annamale Anita ${ }^{6,7,8}$, Alberti Adriana ${ }^{6,7,8}$, Da Silva Corinne ${ }^{6,7,8}$, Wincker Patrick ${ }^{6,7,8}$, \\ Le Crom Stephane ${ }^{1}$, Not Fabrice ${ }^{9,{ }^{*}}$, Bittner Lucie ${ }^{1,{ }^{*}}$
}

${ }^{1}$ Sorbonne Univ, UPMC Univ Paris 06, Univ Antilles Guyane, Univ Nice Sophia Antipolis,CNRS,EPS IBPS, Paris, France.

${ }^{2}$ UPMC, CNRS, FR2424, ABiMS,Stn Biol, Roscoff, France.

${ }^{3}$ UPMC, CNRS, FR2424, Roscoff Culture Collect,Stn Biol Roscoff, PI Georges Teissier, Roscoff,

France.

${ }^{4}$ Natl Inst Water \& Atmospher Res NIWA Ltd, Wellington, New Zealand.

${ }^{5}$ IFREMER, Ctr Brest, DYNECO PELAGOS, Plouzane, France.

${ }_{7}^{6}$ CEA, Inst Genom, GENOSCOPE, Evry, France.

${ }^{7}$ CNRS, UMR8030, Evry, France.

${ }^{8}$ Univ Evry Val dEssonne, Evry, France.

${ }^{9}$ CNRS, UMR 7144, Stn Biol Roscoff, PI Georges Teissier, Roscoff, France.

* Corresponding authors : email addresses : arnaud.meng@etu.upmc.fr ; not@sb-roscoff.fr ; lucie.bittner@upmc.fr

\begin{abstract}
:
Dinoflagellates are one of the most abundant and functionally diverse groups of eukaryotes. Despite an overall scarcity of genomic information for dinoflagellates, constantly emerging high-throughput sequencing resources can be used to characterize and compare these organisms. We assembled de novo and processed 46 dinoflagellate transcriptomes and used a sequence similarity network (SSN) to compare the underlying genomic basis of functional features within the group. This approach constitutes the most comprehensive picture to date of the genomic potential of dinoflagellates. A core-predicted proteome composed of 252 connected components (CCs) of putative conserved protein domains (pCDs) was identified. Of these, 206 were novel and 16 lacked any functional annotation in public databases. Integration of functional information in our network analyses allowed investigation of pCDs specifically associated with functional traits. With respect to toxicity, sequences homologous to those of proteins found in species with toxicity potential (e.g., sxtA4 and sxtG) were not specific to known toxinproducing species. Although not fully specific to symbiosis, the most represented functions associated with proteins involved in the symbiotic trait were related to membrane processes and ion transport. Overall, our SSN approach led to identification of 45,207 and 90,794 specific and constitutive pCDs of, respectively, the toxic and symbiotic species represented in our analyses. Of these, $56 \%$ and $57 \%$,
\end{abstract}


respectively (i.e., 25,393 and 52,193 pCDs), completely lacked annotation in public databases. This stresses the extent of our lack of knowledge, while emphasizing the potential of SSNs to identify candidate $\mathrm{pCDs}$ for further functional genomic characterization.

Keywords : genomics, proteomics, microbial biology, molecular evolution, protists, transcriptomics 


\section{INTRODUCTION}

Dinoflagellates are unicellular eukaryotes belonging to the Alveolata lineage

25 (Bachvaroff et al., 2014). This group encompasses a broad diversity of taxa that have a

26 long and complex evolutionary history, play key ecological roles in aquatic ecosystems,

27 and have significant economic impacts (reviewed in Murray et al. 2016; Janouškovec et

28 al. 2016). The ecological success of dinoflagellates in the marine planktonic environment

29 is assumed to be due to their ability to exhibit various survival strategies associated with

30 an extraordinary physiological diversity (Murray et al., 2016). Nearly half of dinoflagellates

31 have chloroplasts, but most of these are likely mixotrophic, combining photosynthetic and

32 heterotrophic modes of nutrition (reviewed in Jeong et al. 2010; Stoecker et al. 2017).

33 Many dinoflagellates produce toxins and form long-lasting harmful algal blooms with

34 deleterious effects on fisheries or aquaculture (reviewed in Flewelling et al. 2005). Some

35 species of the genus Alexandrium can produce toxins that effect higher trophic levels in

36 marine ecosystems (i.e. copepods, fish) and are harmful to humans (Kohli et al., 2016;

37 Murray et al., 2016; Orr et al., 2013). Members of the genus Symbiodinium are known to

38 establish mutualistic symbioses with a wide diversity of benthic hosts, sustaining reef

39 ecosystems worldwide (Goodson et al., 2001; Lin et al., 2015). Interactions between

40 dinoflagellates and other marine organisms are extremely diverse, including

41 (photo)symbioses (Decelle et al., 2015), predation (Jeong et al., 2010), kleptoplasty (Gast

42 et al., 2007), and parasitism (Siano et al., 2011). Dinoflagellates have been highlighted as

43 important members of coastal and open-ocean protistan communities based on

44 environmental molecular barcoding surveys (Le Bescot et al., 2016; Massana et al., 2015) 
45 and the parasitic syndiniales in particular have been identified as key players that drive in

46 situ planktonic interactions in the ocean (Lima-Mendez et al., 2015).

47 Along with metabarcoding surveys based on taxonomic marker genes, 48 environmental investigations of protistan ecology and evolution involve genomic and

49 transcriptomic data. Interpretation of such large datasets is limited by the current lack of

50 reference data from unicellular eukaryotic planktonic organisms, resulting in a high

51 proportion of unknown sequences (Caron et al., 2016; Sibbald and Archibald, 2017). This

52 is particularly significant for dinoflagellates as this taxon remains poorly explored at the

53 genome level, with only three full genome sequences published so far (Aranda et al., 2016;

54 Lin et al., 2015; Shoguchi et al., 2013). Their genomes are notoriously big (0.5 to 40x

55 larger than the human haploid genome) and have a complex organization (Jaeckisch et

56 al., 2011; Murray et al., 2016; Shoguchi et al., 2013). Consequently, most recent studies

57 investigating functional diversity of dinoflagellates rely on transcriptomic data to probe

58 these non-model organisms.

59 The Moore Foundation Marine Microbial Eukaryotic Transcriptome Sequencing

60 Project (MMETSP, https://www.ncbi.nlm.nih.gov/bioproject/248394, (Keeling et al., 2014))

61 provided the opportunity to produce a large quantity of reference transcriptomic data

62 (Sibbald and Archibald, 2017). Among the 650 transcriptomes released, 56 were from 24

63 dinoflagellate genera encompassing 46 distinct strains (Keeling et al., 2014). This dataset

64 constitutes a unique opportunity to investigate the genomic basis of the major evolutionary

65 and ecological traits of dinoflagellates (Janouškovec et al., 2016). Performing a global

66 analysis of such a large dataset ( $\sim 3$ million sequences) is challenging and requires

67 innovative approaches. Most studies published so far have targeted specific biological

68 processes and pathways, focusing on a small subset of the available data (Dupont et al.,

69 2015; Kohli et al., 2016; Meyer et al., 2015). In one recent study a 101-protein dataset was

70 used to produce a multiprotein phylogeny of dinoflagellates (Janouškovec et al., 2016). As 
71 a large fraction of the sequences produced in the MMETSP project do not have any distant

72 homologues in current reference databases, almost half $(46 \%)$ of the data remains

73 unannotated.

74 With the advent of high-throughput sequencing technologies and its inherent massive

75 production of data, sequence similarity network (SSN) approaches (Atkinson et al., 2009;

76 Cheng et al., 2014; Méheust et al., 2016) offer an alternative to classical methods,

77 enabling inclusion of unknown sequences in the global analysis (Forster et al., 2015;

78 Lopez et al., 2015). In a functional genomic context, SSNs facilitate large-scale

79 comparison of sequences, including functionally unannotated sequences, and hypothesis

80 design based on both model and non-model organisms. For instance, SSN has been used

81 to define enolase protein superfamilies and assign function to nearly $50 \%$ of sequences

82 composing the superfamilies that had unknown functions (Gerlt et al., 2012). Here we

83 used a SSN approach involving 42 de novo assembled transcriptomes from the MMETSP

84 project as well as new transcriptomes of four recently described dinoflagellates to unveil

85 the core-, accessory-, and pan-proteome of dinoflagellates and to define gene sets

86 characteristic of selected functional traits.

\section{RESULTS}

\section{Dataset metrics overview}

89 A total of 46 transcriptomes were assembled and retained for further analyses

90 using our protocol and a proteome was predicted for each transcriptome (Tab. 1). Globally,

91 more than half of the protein-coding domains matched with functional annotations in

92 InterPro (58\%: 746,074 of $1,275,911)$ of which 549,459 had an identified Gene Ontology

93 (GO) annotation. All individually assembled transcriptomes, derived proteomes and their 
94 corresponding functional annotations available at

95 https://figshare.com/projects/Dinoflagellate_SSN/28410.

96 Our SSN involves 1,275,911 vertices (protein-coding domains or, for short

97 thereafter, domains) linked by $6,142,013$ edges (pairwise sequence identity value $\geq 60 \%$ ).

98 The network consisted of 350,267 connected components (CCs) with 11,568 of these

99 having a size from 10 to 100 vertices. It encompassed 46 proteomes having a mean of

10060,661 domains with an average length of $307 \mathrm{bp}$. According to InterPro functional

101 annotations, $50.5 \%$ of the CCs were composed of unannotated sequences only.

102 Identification of core / accessory / pan connected components

103 Global comparison analysis has been processed on 43 of the 46 proteomes that

104 have a comparable number of domains. The analysis revealed 252 core CCs, 160,431

105 accessory CCs, and 347,551 pan CCs (Fig. 1A). The trend of the core proteome CC

106 number was extrapolated using a non-linear regression model. The best-fit function was

$107 y=a / x$, with $y$ the predicted number of core CCs, $x$ the number of proteomes and $a$ an

108 estimated parameter. For 2 to 43 proteomes, this model had a Pearson correlation

109 coefficient of 0.97 ( $p$-value of estimated parameter $a<2 e-16$ ). The number of core CCs

110 for 50, 60 and 70 proteomes was extrapolated to 170, 144 and 123 CCs respectively,

111 without displaying a saturation to a fixed number of core CCs. The Pielou diversity indices

112 shew a mean value of 0.96 , indicating the core CCs were evenly structured, i.e. rarely

113 being dominated by a single proteome.

114 Functional annotation revealed that $91,4 \%$ of core domains matched to the

115 InterPro database. According to GOslim functional categories, the most abundant

116 annotations correspond to "ribosomal proteins" having a role in RNA translation (i.e. 7,968

117 of 37,842 core domains) followed by protein involved in phosphorylation, in signal

118 transduction and in cell redox homeostasis (Fig. S2). The 37,842 core domains were

119 further analyzed by comparison to other reference databases: the proportion of matches 
120 reached 12.5\% (involved in 51 CCs) against BUSCO (Simão et al., 2015), 79.6\% (involved

121 in $190 \mathrm{CCs}$ ) against UniProtKB/Swiss-Prot and 93.7\% (involved in $236 \mathrm{CCs}$ ) (Simão et al.,

122 2015) against nr (Fig. 1B). 16 CCs (i.e. 946 domains) did not have any match (Fig. 1B).

123101 orthologous alignments used for a recent phylogeny (Janouškovec et al., 2016) were

124 compared to the core domains : 1606 domains from 46 CCs matched with at least one of

125 the 101 alignments (Fig. S3, Tab. S15), but no homology was found with the domains from

126 our 16 unknown core CCs.

\section{Dinoflagellate functional traits investigations}

128 In the SSN based on the 46 proteomes, the number of CCs exclusively composed

129 of domains from species tagged with a single functional trait (trait-CCs) has been reported

130 for each trait (Tab. S1-S9), as well as the percentage of trait-CCs (e.g. trait-CC including

131 at least one InterPro functional annotation). As expected considering the taxonomic

132 coverage of our dataset, a maximum number of trait-CCs were found for the "chloroplast"

133 trait (336,099 CCs) whereas a minimum number was found for the "parasitism" trait (826

134 CCs). The "chloroplast" trait had the highest percentage of annotated trait-CCs (93\%)

135 while the "parasitism" trait had the lowest (23\%) (Fig. S4). Among the trait-CCs, a total of

1365 "toxicity potential" trait-CCs involving 7 of 14 possible proteomes were detected.

137 Likewise, 2 "symbiosis" trait-CCs including 8 of 12 possible proteomes were identified

138 (Tab. S4 \& S6).

139 Focus on the "toxicity potential" functional trait

140 Well-described proteins involved in dinoflagellate toxicity, the polyketide synthases

141 (PKS) and saxitoxins (STX) were sought within our dataset. 36 PKS homologs were

142 identified in 17 "toxicity potential" trait-CCs (composed of 45 domains) (Tab. S10) whereas

143646 PKS homologs were found in 165 non-"toxicity potential" CCs (composed of 1,144

144 domains). The 1,189 corresponding domains (i.e. $45+1,144$ ) had either a Thiolase-like

145 functional annotation (1,159 domains), which corresponds to the superfamily of KS 
146 enzyme domains of PKS, or lacked annotation (30 domains) according to the InterPro

147 database. The sxtA4 and sxtG genes have been reported to be found in potentially toxic

148 species. No stxA4 or stxG (i.e. genes associated with saxitoxin producing species (Stüken

149 et al., 2011)) homolog was found in "toxicity potential" trait-CCs (Tab. S11). In contrast, 4

150 homologs of stxA4 and 3 homologs of stxG were identified in non-"toxicity potential" trait-

151 CCs. sxtA4 hits correspond to 1 CCs (composed of 6 domains), and sxtG hits belonged

152 to $1 \mathrm{CC}$ composed of 3 domains. The 4 sxtA4 homologs matched the InterPro annotation

153 "pyridoxal phosphate-dependent transferase" and the 2 remaining domains of the CC

154 were unannotated. A single InterPro annotation was found for the CC composed by sxtG

155 homologs and corresponded to an amidinotransferase known as a sxtG protein domain

156 (Tab. S11).

157 GO functional annotations of all "toxicity potential" trait-CCs revealed that the 158 cellular component functional level, "membrane" and "integral component of membrane",

159 annotations represented $51 \%$ and $27 \%$ of the domains respectively (Fig. $2 A$ ). At the

160 biological process annotation level, 14\% of the domains were linked to "ion transport" (Fig.

1612 2). At the molecular function annotation level, $24 \%$ corresponded to "protein binding"

162 (Fig. 2A). Differential composition of functional annotations between proteomes revealed

163 that "ion transport" protein domains occurred 7 times more often in "toxicity potential" trait-

164 CCs whereas pentatricopeptide repeat, C2 domain, P-loop containing nucleoside 165 triphosphate hydrolase, Pyrrolo-quinoline quinone beta-propeller repeat, Quinonprotein 166 alcohol dehydrogenase-like and Thrombospondin type 1 repeat domains occurred 1 to 2 167 times more often in "toxicity potential" trait-CCs (Fig. 2B).

168 CCs involving most toxic representatives were investigated to reveal functions 169 shared among toxic species only (Fig. 2C). Five core "toxicity potential" trait-CCs 170 (corresponding to a total of 49 domains) encompassed 7 of the 14 toxic dinoflagellate 171 proteomes considered in our analysis. Not a single of these 49 domains had a GO 
172 annotation. Based on InterPro annotations, 3 of the 5 CCs are respectively composed of

17314 "nucleotide-binding alpha-beta plait" domains, 7 "P-loop containing nucleoside

174 triphosphate hydrolase" domains and 8 "nucleotide-diphospho-sugar transferase"

175 domains. The remaining two of these 5 CCs were entirely composed of 7 and 15

176 unannotated domains. Supplementary results about the taxonomic and functional

177 composition of the core "toxicity potential" trait-CCs can be found on

$178 \mathrm{https}: / /$ figshare.com/projects/Dinoflagellate_SSN/28410.

179 Among the 45,207 "toxicity potential" trait-CCs, $69 \%$ of them (i.e. 31,496 CCs

180 corresponding to 70,359 domains) completely lacked InterPro functional annotations.

181 Additional alignments to the $\mathrm{nr}$ database (using an e-value of $1 \mathrm{e}-3$ and a sequence identity

182 higher than $80 \%$ ) revealed 6,103 hits including 283 domains, which finally lowered the

183 number of "toxicity potential" trait-CCs without functional annotation to 25,393 .

184 Focus on the "symbiosis" functional trait

185 A large range of dinoflagellates, expresses genes identified in the literature as 186 potentially involved in symbiotic processes (Tab. S12). 150 of these gene sequences were 187 sought in our datasets. 8 domains from 5 "symbiosis" trait-CCs were identified as proteins 188 involved in symbiosis establishment (nodulation protein nolO and phosphoadenosine 189 phosphosulfate reductase), cell recognition processes (merozoite surface protein), and 190 highlighted in cnidarian-algal symbiosis (peroxiredoxin, ferritin) (Tab. S12). Similarly, 71

191 domains (spread across $21 \mathrm{CCs}$ ) were found in non-"symbiosis" trait-CCs. Functions of 192 these 71 domains are involved in symbiosis establishment (P-type H+-ATPase, 193 phosphoadenosine phosphosulfate reductase), cell recognition processes (merozoite 194 surface protein 1) and exposed in cnidarian-algal symbiosis (superoxide dismutase, 195 catalase, peroxiredoxin, glutathione peroxidase, g-glutamylcysteine synthetase).

196 GO functional annotations from all "symbiosis" trait-CCs (Fig. 2D) revealed that at 197 the cellular component level, $83 \%$ of the annotations were "membrane proteins". At the 
198 biological process level, $21 \%$ of the annotations were "ion transport" domains and $18 \%$

199 were involved in "protein phosphorylation". At the molecular function level, $39 \%$ of the

200 annotations were "protein-binding" domains, 10\% were involved in "ion channel activity"

201 and $9.9 \%$ in "calcium ion binding". Differential composition of functional annotations

202 between proteomes revealed 4 annotations occurring 2 to 10 times more in symbiotic

203 lineages: ion transport, ankyrin repeat, EF-hand and zinc finger, and CCCH-type (Fig. 2E).

204 Two core CCs of the "symbiosis" trait involving a maximum of 8 distinct proteomes

205 and 187 core "symbiosis" trait-CCs involving 7 proteomes (of the 12 proteomes symbiotic

206 species available) were identified (Fig. 2F). GO annotations of these 189 core "symbiosis"

207 trait-CCs revealed that the majority of the domains (i.e. 1400 out of 1896) could not be

208 functionally annotated. Among those that could be annotated, $73.8 \%$ of the domains

209 corresponded to "membrane proteins" (cellular component), and the remainder

210 corresponded to "proteins of photosystem I", "extracellular region" and "spliceosomal

211 complex". With respect to biological process, $31.9 \%$ of the domains were involved in ion

212 transport while $23.8 \%$ were involved in proteolytic processes (Tab. S13). Supplementary

213 results about the taxonomic and functional composition of the core "symbiosis" trait-CCs

214 can be found on https://figshare.com/projects/Dinoflagellate_SSN/28410.

215 Among the 90,794 "symbiosis" trait-CCs, 57\% of them (i.e. 52,491 CCs corresponding to

216 130,673 domains) completely lacked InterPro functional annotations. Additional

217 alignments to the $\mathrm{nr}$ database (using an e-value of $1 \mathrm{e}-3$ and a sequence identity higher

218 than $80 \%$ ) revealed matches for 495 domains, which finally lowered the number of "toxicity

219 potential" trait-CCs without functional annotation to $52,193$. 


\section{DISCUSSION}

\section{An efficient analysis pipeline to study non-model organisms and their dark matter}

222 Our de novo assembly and downstream pipeline analysis of multiple dinoflagellate

223 transcriptomes overcame several biases inherent to de novo assembly processes (Fig.

224 S5). For instance, the domain prediction step selected transcripts involving ORFs and

225 protein domains and allowed removal of truncated or chimeric transcripts (Yang and

226 Smith, 2013). Data derived from high quality transcriptomes (cf. definition in the Material

227 and Methods section) enabled construction of sequence similarity networks to focus on

228 shared domains among multiple proteomes. Considering our 46 proteomes, a mean value

229 of 60,661 domains was found, which is consistent with the previously estimated range of

23034,156 to 75,461 genes in dinoflagellates (Murray et al., 2016). The median length of the

231 domains was $307 \mathrm{bp}$, also consistent with the median protein length of $361 \mathrm{bp}$ from

232 genomes of 5 model species (Homo sapiens, Drosophila melanogaster, Caenorhabditis

233 elegans, Saccharomyces cerevisiae and Arabidopsis thaliana) (Brocchieri and Karlin, 234 2005).

235 Sequence similarity networks represent an informative and pragmatic way to study

236 massive datasets (Alvarez-Ponce et al., 2013; Atkinson et al., 2009; Cheng et al., 2014;

237 Forster et al., 2015; Méheust et al., 2016). In (Cheng et al., 2014), 84 genome-derived

238 proteomes of prokaryotes (i.e. 128,628 sequences) were used to study the impact of redox

239 state changes on their gene content and evolution. The authors found that the core CCs

240 revealed a correlation between their network structure and differences in respiratory

241 phenotypes. Our SSN has allowed simultaneous exploration of 46 transcriptome-derived

242 proteomes $(1,275,911$ sequences), including their overwhelming "dark matter" (i.e. here

243 domains totally lacking functional annotation). High identity and coverage threshold values

244 used to filter alignments ensured that only high quality alignments were included in the 
245 network (Bittner et al., 2010). The integration of 4 new dinoflagellate proteomes

246 represented an increase of $14 \%$ of domains in the SSN and overall the dataset represents

247 the most comprehensive picture to date of the genomic potential of dinoflagellates. This

248 new resource and comparative genomic approach allow generation and testing of original

249 hypotheses about the genomic basis for evolutionary history and life style, functional traits,

250 and specificities of dinoflagellates.

251 Large-scale comparison of dinoflagellate proteomes confirms the extent of our

252 lack of knowledge

253 The SSN analyses allowed characterization of the core and accessory proteomes

254 for this large dataset of non-model organisms. Because our analysis relied on a de novo

255 assembled, transcriptome-derived, proteome SSN rather than classical knowledge-based

256 genomics, it also promoted discovery of new CCs, each of which can be functionally

257 assimilated to a single putative conserved protein-domain ( $p C D)$ in such non-model

258 organisms (Lopez et al., 2015) (Fig. S6).

259 The core dinoflagellate proteome identified in our analysis was composed of 252

260 pCDs (Fig. 1A), a size that falls in the range of the latest estimates for bacteria (352 core

261 genes) (Yang et al., 2015) and eukaryotes (258 core genes in CEGMA, and more recently

262429 single-copy orthologs in BUSCO) (Parra et al., 2007; Simão et al., 2015). The

263 extrapolation of the number of core CCs does not saturate, suggesting that the number of

264 core CCs for dinoflagellates could be less than 256. Our comparative analysis with the

265 most up-to-date eukaryotic orthologous gene database BUSCO strongly stresses the

266 need to generate more gene and protein data for non-model marine organisms in order to

267 populate reference databases (Armengaud et al., 2014). The small overlap between core 
268 dinoflagellate $\mathrm{pCDs}$ and the BUSCO database suggests that essential functions 269 expressed by dinoflagellates are distantly related to those of current model eukaryotes.

270 Our SSN constitutes a strong basis for exploration and refinement of functional 271 annotations as our dataset encompassed a broad range of dinoflagellate taxa according 272 to recent phylogenetic analyses (Bachvaroff et al., 2014; Janouškovec et al., 2016).

273 However, the identified core proteome can only be considered partial as our dataset i- did 274 not include representatives of all described dinoflagellate lineages, and ii- relied on 275 transcriptomic (i.e. gene expression) data that can vary according to eco-physiological 276 conditions and/or life-cycle stage. The content of our SSN can be however updated 277 permanently to refine these estimates as new dinoflagellate genomic data are 278 accumulated (Aranda et al., 2016; Lin et al., 2015; Shoguchi et al., 2013). 236 (93\%) core 279 CCs involving one or more functionally annotated domains (Fig. 2B) can be exploited to 280 extend annotation to other aligned domains within each CC. For instance, looking for the 281 HSP70 conserved protein domain, which is ubiquitous in all eukaryotic organisms (Germot 282 and Philippe, 1999), 320 domain annotated as HSP70, all belonging to a single CC 283 composed of 328 domain. The 8 remaining domain sequences were either imprecisely 284 annotated as chaperone DnaK (1 sequence), cyclic nucleotide-binding domain (2 285 sequences), heat shock protein 70 family (3 sequences) or annotation was simply missing 286 (2 sequences) (Tab. S14). As HSP70 represented $97 \%$ of the annotations, it is reasonable 287 to extend it to all sequences forming the CC. Considering only CCs that were at least half 288 composed of annotated domain sequences, this approach could be applied to 289 complement the functional characterization of 49 CCs (583 unannotated domains).

290 Janouškovec et al., 2016 used for the first time a multi-protein dataset providing a 291 robust phylogeny for dinoflagellates. The comparison of the 101 orthologous alignments 292 (Janouškovec et al., 2016) with our 252 pCDs revealed that 206 of them could constitute 
293 good new candidates for refining dinoflagellate phylogeny, increasing by nearly $200 \%$ the

294 quantity of information available for such studies.

295 Among the 176,958 distinct CCs entirely composed of unannotated domains, 16

296 CCs or pCDs (composed of 946 domain) belonged to our core dinoflagellate proteome

297 (Fig. 1B). This highlights that many fundamental genomic features remain to be

298 characterized in this lineage. These unknown groups of homologous domains are

299 excellent potential candidate markers to further investigate dinoflagellate genomics at a

300 broad scale and might also be useful for identification of dinoflagellates within complex

301 environmental genomic datasets.

\section{Confirmation and the new insights about the genomic bases of the toxicity}

303 Toxic dinoflagellates represent about $80 \%$ of toxic eukaryotic phytoplankton

304 species (Janouškovec et al., 2016). Production of toxins by dinoflagellates is well known

305 and can cause major health and economic problems. Karenia brevis, for example, is

306 known to produce brevetoxins which cause fish mortality and can affect human health

307 through the consumption of contaminated seafood or direct exposure to harmful algal

308 blooms (HABs) (Flewelling et al., 2005). To date, several dinoflagellate toxins have been

309 chemically and genetically characterized (Cusick and Sayler, 2013; Kellmann et al., 2010;

310 Stüken et al., 2011; Wang, 2008). In our SSN analyses, PKS homologs were identified in

311 CCs composed of domains from both "toxicity potential" and non-"toxicity potential"

312 species. This result validates a previous report that PKS proteins are not exclusive to toxic

313 species ), but are in fact involved in the production of a variety of natural products such as

314 small acids, acetyl-CoA or propionyl-Co (Khosla et al., 2014). Spreading information

315 among unannotated domains in both "toxicity potential" and non-"toxicity potential" trait-

316 CCs in which PKS were identified allowed extension of the potential PKS-like annotation

317 to 9 and 498 domains respectively (Tab. S15). PKS domains for 4 extra species

318 (Alexandrium catenella, Kryptoperidinium foliaceum, Protoceratium reticulatum and 
319 Crypthecodinium cohnii) were also detected compared to the database from (Kohli et al., 320 2016) (Tab. S13) (Kohli et al., 2016).

321 With respect to saxitoxin production, as no sxtA4 and sxtG (i.e. the combination of 322 genes associated with saxitoxin producing species (Stüken et al., 2011)) homologs were

323 found in "toxicity potential" trait-CCs, it suggests that such proteins are also not exclusively

324 expressed by toxic species and/or are not constitutively expressed. As Murray et al., 2015,

325 we detected sxtA4 and sxtG proteins in the transcriptomes of the toxic species Pyrodinium

326 bahamense and Gymnodinium catenatum (Tab. S11). However, our results also differed

327 somewhat from this previous study even if it is based on the same initial MMETSP dataset.

328 Specifically, we were not able to detect sxtA4 in Alexandrium fudyense (Murray et al.,

329 2015) whereas sxtA4 domains were detected Pelagodinium beii (Murray et al., 2015) (Tab.

330 S11). These differences may be due to the use of distinct de novo assembly tools and

331 pCD prediction processes, illustrating the requirement to ultimately combine in vitro and

332 in silico methods in order to unambiguously characterize toxic species. We also confidently

333 detected 1 sxtA4 homolog and 1 sxtG homolog in $P$. beii, an a priori non-toxic species that

334 has never been reported as a STX-producer. sxtG has previously been identified in non-

335 toxic species (Orr et al., 2013), but the presence of both domains (sxtA4 and sxtG) in a 336 non-toxic species would be a first recorded discovery. If this pattern would not be

337 confirmed in the future by in silico and in vitro analyses, such result might be a 338 consequence from a contamination. MMETSP transcriptomes contaminations is

339 furthermore a recurrent debate in the protistology community (e.g. Dorrell et al., 2017),

340 however as our SSN vertices are labelled with the taxonomy and the strain names, it is

341 possible and easy, whenever one decides that a strain is doubtful, to remove its

342 corresponding vertices and edges. From an evolutionary point of view, as PKS and STX

343 genes are also found in species currently described as non-toxic, it seems that like for

344 snake venoms, dinoflagellate toxins might have evolved by recruitment of genes encoding 
345 regular proteins followed by gene duplication and neo-functionalization of the domains

346 (Vonk et al., 2013).

347 Composition of "toxicity potential" trait-CCs showed that membrane protein and 348 more specifically ion transport proteins are important components of toxic species. This is

349 in agreement with that ion channel proteins and proteins involved in neurotransmission

350 are mediators of dinoflagellate toxicity (Cusick and Sayler, 2013; Wang, 2008). Finally, 2

351 of the 5 CCs with the most toxic representatives (i.e. 7 species) were exclusively

352 composed of unannotated domains, representing essential functions constitutively

353 expressed by toxic species only and for which further investigations are required to better

354 characterize toxic dinoflagellates.

\section{From the study of symbiosis to the detection of genomic markers}

356 The "symbiotic" gene set compiled from the literature based on their involvement

357 in the establishment and maintenance of symbiosis (Lehnert et al., 2014; Lin et al., 2015)

358 was found here in both "symbiosis" trait-CCs and in non-"symbiosis" trait-CCs (Tab. S12),

359 suggesting that these proteins are constitutively expressed by all dinoflagellate species.

360 This result may reflect the fact that the transcriptomes of dinoflagellate strains were not

361 directly isolated from symbiotic conditions, but rather from their free-living stages

362 maintained in culture. Symbiotic genes identified from the literature were originally inferred

363 from studies on holobionts (i.e. host and symbionts) but proved here not to be exclusive

364 to symbiotic dinoflagellates when performing global comparison of multiple datasets.

365 Functional annotations of "symbiosis" trait-CCs revealed an overall clear

366 domination of proteins involved in phosphorylation and ion transport domains (e.g.

367 sodium, potassium and calcium ion channel proteins) located within membrane

368 compartments (Fig. 2D). The 4 most prominent functions that occurred 2 to 10 times more

369 often in "symbiosis" trait-CCs (Fig. 2E) were related to ion transport domains and

370 regulation processes. Protein phosphorylation is known to take part in cellular 
371 mechanisms in response to the environment (Day et al., 2016) and play a key role in signal

372 transduction to other cells in plant parasitism and symbiosis models (Lionetti and Metraux,

373 2015). The specific dominant presence of ion transport domains (also involved in cell

374 signalling and cell adaptation to the environment) in symbiotic dinoflagellates could

375 represent a constitutive characteristic of symbiotic species facilitating establishment and

376 maintenance of the symbiosis. Notably, the role of ion channel proteins has been

377 highlighted as essential in plant root endosymbiosis (Charpentier et al., 2008; Matzke et

378 al., 2009). This suggests that symbiotic species are likely to be constitutively better

379 adapted for environmental adaptations.

$38045 \%$ of the domains associated to symbiotic species were unknown (Tab. S16)

381 and 129,754 domains from 52,193 "symbiosis" trait-CCs remained unannotated according

382 to the InterPro and nr databases. The 2 "symbiosis" trait-CCs encompassing 8 distinct

383 species were exclusively composed of unannotated domains, suggesting that they

384 represent $\mathrm{pCD}$ with fundamental, yet unknown, functions constitutively expressed by

385 symbiotic species. Overall, our analyses demonstrate that SSN has significant potential to

386 reveal the variety of annotated and unknown pCDs that constitute good candidates for

387 further study to characterize and understand the genomic basis of symbioses involving 388 dinoflagellates.

\section{CONCLUSION}

$390 \quad$ Our efficient analysis pipeline and our innovative analysis strategy allowed us to 391 study the genomic of non-model organisms, here dinoflagellates, and their dark matter on

392 a massive scale. We confirmed that genes currently listed as implied in the "toxicity

393 potential" or "symbiosis" functional traits, were not specific from toxic or symbiotic lineages,

394 thus implying that these sequences have evolved by recruitment of genes encoding 395 regular proteins followed by gene duplication and neo-functionalization of these domains. 
396 By contrast, our approach, also identified candidate putative conserved protein domains

397 for further genomic characterization of these functional traits. These markers are to date

398 working hypotheses which will have to be further confirmed by future molecular studies (at

399 the bench using more samples and differential expression analyses, PCR and qPCR), and

400 also by mining directly environmental meta-omics datasets.

401

402 M\&M

403 Dataset building

404 The dataset used in our study included all dinoflagellate transcriptomes available 405 in the MMETSP project repository (https://www.ncbi.nlm.nih.gov/bioproject/248394) as 406 well as 4 transcriptomes generated for this study (more details in the following section) 407 (Fig. S7). This represented a total of 60 datasets (Fig. S7). Two Pelagodinium beii 408 RCC1491 datasets appeared (one produced by the MMETSP, and one produced in the 409 framework of our analysis), we nevertheless analysed them separately as sequencing 410 experiments were performed in distinct institutes (cf. recommendations in Keeling et al 411 2014). Furthermore, transcriptomes from the same species but produced from different 412 strains were pooled when the number of reads were insufficient to create two 413 transcriptomes of "high quality" (n.b. a definition of the "high quality" transcriptome is given 414 a few lines below) if the sequencing experiments were performed in the same institute. 415 Consequently, the two Oxyrrhis marina strains (NA and LB1974), the two Prorocentrum 416 minimum strains (CCMP1329 and CCMP2233) and the two Polarella glacialis strains 417 (CCMP1383 and CCMP2088) were pooled; whereas we did not pool the Brandtodinium 
418 nutricula (RCC3387 and RCC3468) which were involving both enough reads to perform

419 reliable assemblies.

$420 \quad$ These 60 datasets (Fig. S7) correspond to 48 distinct species from 34 genera, 18

421 families, and 11 of the 21 current dinoflagellate taxonomic orders according to the

422 taxonomic framework of the WoRMS database (http://www.marinespecies.org/index.php)

423 and Algaebase database (Guiry and Guiry, 2018). Taxonomy and functional traits

424 information (i.e. chloroplast occurrence and origin, trophic mode, toxicity potential, ability

425 to live in symbiosis, to perform kleptoplasty, to be a parasite or to be toxic for fauna) were

426 indicated for each organism (Fig S7).

427 Cultivation and RNA sequencing for four dinoflagellate strains

$428 \quad$ Free-living clonal strains of the dinoflagellate species Brandtodinium nutricula

429 (RCC3468) (Probert et al., 2014) and Gymnoxanthella radiolariae (RCC3507) (Yuasa et

430 al., 2016) isolated from symbiotic Radiolaria, Pelagodinium beii (RCC1491) (Siano et al.,

431 2010) isolated from a foraminiferan host, and the non-symbiotic Heterocapsa sp.

432 (RCC1516) were obtained from the Roscoff Culture Collection (www.roscoff-culture-

433 collection.org). Triplicate $2^{-\mathrm{L}}$ acid-washed, autoclaved polycarbonate Nalgene bottles were

434 filled with 0.2 micron filter-sterilized (Stericup-GP, Millipore) seawater with K/2 (-Tris,-Si)

435 medium supplements (Keller et al., 1987) and inoculated with an exponentially growing

436 culture of each strain. All cultures were maintained at $18^{\circ} \mathrm{C}, \sim 80 \mu \mathrm{mol}$ photon $\mathrm{m}^{-2} \mathrm{~s}^{-1}$ light

437 intensity and 14:10 light:dark cycle. Cell abundance was monitored daily by flow cytometry

438 with a FACSAria flow cytometer (Becton Dickinson, San José, CA, USA) and derived cell

439 division rates were used to monitor the growth phase of the culture. Light and dark phase

440 samples for transcriptome analyses were taken from exponential and stationary phase

441 cultures. $100 \mathrm{~mL}$ aliquots from each culture were filtered onto 3 micron pore-size

442 polycarbonate filters with an autoclaved $47 \mathrm{~mm}$ glass vacuum filter system (Millipore) and

443 a hand-operated PVC vacuum pump with gauge to maintain the vacuum pressure below 
$4445 \mathrm{~mm} \mathrm{Hg}$ during filtration. The filter was then placed in a sterile $15 \mathrm{~mL}$ falcon tube filled 445 with ca. $5 \mathrm{ml} \mathrm{TriZol} \mathrm{and} \mathrm{stored} \mathrm{at}-80^{\circ} \mathrm{C}$.

446 Total RNA was purified directly from the filters stored in TriZol using the Direct-zol

447 RNA Miniprep kit (ZymoResearch, Irvine, CA). First, the tube containing the filter 448 immersed in TriZol was incubated for $10 \mathrm{~min}$ at $65^{\circ} \mathrm{C}$. Then, after addition of an equal 449 volume of $100 \%$ EtOH and vortexing, the mixture was loaded into a Zymo-SpinllC column 450 and centrifuged for $1 \mathrm{~min}$ at $12,000 \mathrm{~g}$. The loading and centrifugation steps were repeated 451 until exhaustion of the mixture. RNA purification was completed by prewash and wash 452 steps following the manufacturer's instructions and RNA was directly eluted in $45 \mu \mathrm{L}$ 453 nuclease-free water. The in-column DNAse step was replaced by a more efficient post454 extraction DNAse treatment using the Turbo DNA-free kit (Thermo Fisher Scientific, 455 Waltham, MA) according to the manufacturer's rigorous DNase treatment procedure. After 456 two rounds of 30 minutes incubation at $37^{\circ} \mathrm{C}$, the reaction mixture was purified with the 457 RNA Clean and Concentrator-5 kit (ZymoResearch) following the procedure described for 458 retention of $>17 \mathrm{nt}$ RNA fragments. Total RNA, eluted in $20 \mu \mathrm{L}$ nuclease-free water, was 459 quantified with RNA-specific fluorimetric quantification on a Qubit 2.0 Fluorometer using 460 Qubit RNA HS Assay (ThermoFisher Scientific). RNA quality was assessed by capillary 461 electrophoresis on an Agilent Bioanalyzer using the RNA 6000 Pico LabChip kit (Agilent 462 Technologies, Santa Clara, CA).

463 RNA-Seq library preparations were carried out from $1 \mu \mathrm{g}$ total RNA using the 464 TruSeq Stranded mRNA kit (Illumina, San Diego, CA), which allows mRNA strand 465 orientation. Briefly, poly(A)+ RNA was selected with oligo(dT) beads, chemically 466 fragmented and converted into single-stranded cDNA using random hexamer priming. 467 Then, the second strand was generated to create double-stranded cDNA. Strand 468 specificity was achieved by quenching the second strand during final amplification thanks 469 to incorporation of dUTP instead of dTTP during second strand synthesis. Then, ready-to- 
470 sequence Illumina libraries were quantified by GPCR using the KAPA Library

471 Quantification Kit for Illumina libraries (KapaBiosystems, Wilmington, MA), and library

472 profiles evaluated with an Agilent 2100 Bioanalyzer (Agilent Technologies). Each library

473 was sequenced using 101 bp paired-end read chemistry on a HiSeq2000 Illumina

474 sequencer.

475 Data filtering and de novo assembly

$476 \quad$ Using Trimmomatic (Bolger et al., 2014), reads with quality below 30 Q on a sliding

477 window size of 10 were excluded. Remaining reads were assembled with the de novo

478 assembler Trinity version 2.1.1 (Grabherr et al., 2011) using default parameters for the

479 paired reads method (strand-specific read orientation RF). Of the initial 60 transcriptome

480 datasets (56 from the MMETSP repository and 4 produced in this study), 57 were

481 successfully assembled. The assembly process could not be completed properly for 3

482 datasets due to a computation error from the assembly software (Karenia brevis strain

483 CCMP 2229, Wilson SP1 and SP3 as a combined assembly, Oxyrrhis marina strain

484 CCMP1795 and Symbiodinium kawagutii strain CCMP2468). Assembled transcripts were

485 then evaluated based on: (i) sequence metrics, and (ii) read remapping rates calculated

486 respectively with homemade scripts and Bowtie 2 in local mode (Langmead et al., 2009)

487 (Tab. 1). Two classes of assembly quality were defined: those with $>30,000$ transcripts

488 with a N50 > $400 \mathrm{bp}$ and read remapping rate $>50 \%$ were tagged as "high quality"

489 transcriptomes whereas the remainders were tagged as "low quality" transcriptomes. An

490 exception was made for one poor quality transcriptome corresponding to the species

491 Oxyrrhis marina (LB1974 and NA strain) composed of 18,275 assembled transcripts that

492 was intentionally tagged as a "high quality" transcriptome because this basal species holds 
493 a key evolutionary and ecological position among dinoflagellates (Bachvaroff et al., 2014;

494 Lee et al., 2014; Montagnes et al., 2011).

495 Coding domain prediction and functional annotation

$496 \quad$ For each transcriptome, coding domain prediction of assembled transcripts was

497 conducted with Transdecoder version 2.0.1 (Haas et al., 2013) to obtain peptide

498 sequences of corresponding domains. We defined each set of predicted protein domains

499 as a proteome. The optional step of Transdecoder consisting in the identification of ORFs

500 in the protein domain database Pfam was not executed in order to avoid a comparative

501 approach that would result in a limited discovery of new sequences. The predicted coding

502 domains were then processed with the InterProScan 5 functional annotation program

503 version 5.11-51.0 (Jones et al., 2014) to scan for protein signatures. Default parameters

504 were used to obtain each proteome. Finally, to get a broad overview of the ontology

505 content of our datasets, GO slims were retrieved from the Gene Ontology Consortium to

506 build a summary of the GO annotations without the detail of the specific fine-grained terms

507 (http://geneontology.org/page/go-slim-and-subset-guide).

508 Sequence similarity network building and exploration

509 A sequence similarity network (SSN) is a graph in which vertices are genomic

510 sequences and the edges represent similarity between sequences. A SSN is composed

511 of connected components (CC) (subgraphs or subnetworks, including at least two vertices

512 disconnected from other subgraphs in the total network). As information can be linked to

513 sequences (e.g. in our study: taxonomy, functional annotation, functional traits), the SSN

514 and its structure can be explored accordingly. Using predicted protein domain sequences,

515 a SSN was constructed with the BLASTp alignment method (Altschul et al., 1990) with an 
516 e-value of 1e-25 using the DIAMOND software (Buchfink et al., 2015). Similarities

517 satisfying query and subject sequence coverages higher than $80 \%$ were kept.

518 Whenever domains aligned together forming a CC it can be assumed that they

519 potentially share a similar molecular function (Marchler-Bauer et al., 2005) and form

520 putative conserved domains ( $\mathrm{pCDs}$ ). SSN exploration and analyses were performed using

$521 \mathrm{R}$ (version 3.2.3) personal scripts and functions implemented in the igraph $\mathrm{R}$ package

522 (version 1.0.1) (Csárdi and Nepusz, 2006). Biological information related to the species

523 considered were mapped on each vertex, and missing information were marked as $\langle N A>$.

524 All scripts and the SSN (as well as the information linked to each vertices) can be found

525 on https://figshare.com/projects/Dinoflagellate_SSN/28410.

526 In our approach, CC number, structure and composition were impacted when edge

527 sequence identity cut off was shifted. We thus tested different similarity thresholds and

528 chose an optimal threshold according to the two following criteria: maximizing the number

529 of large CCs (i.e. minimum of 30 vertices) and the number of CCs involving a single

530 homogeneous functional annotation (i.e. a unique GOslim term at the Biological Process

531 level). An optimal sequence identity threshold at $60 \%$ similarity with our dataset was

532 inferred (Fig. S1). As a last filtering step, we chose to consider only vertices and edges of

533 proteomes that fitted the optimal threshold defined above (Fig. S7), which resulted in a

534 dataset of 46 proteomes (Tab. 1).

53543 proteomes composed of comparable numbers of protein domains (i.e. a 536 minimum of 9,000 domains) (Fig. S8) were used to define the core-, accessory- and pan-

537 proteomes. The core-proteome corresponds to the CCs composed of sequences from

538 every single proteome considered, whereas the accessory-proteome corresponds to the

539 CCs composed of sequences from a single proteome. The pan-proteome corresponds to

540 the total number of CCs identified in the network. To build Fig. 1, proteomes were

541 compared from the largest to the smallest: the two biggest datasets were first selected to 
542 calculate the core/accessory/pan values; then the biggest remaining dataset was added

543 to calculate the core/accessory/pan values for 3 proteomes. etc. until considering the

544 comparison of all 43 proteomes. In addition to the InterProScan annotation process,

545 sequences belonging to core CCs were compared to 3 databases: (i) the BUSCO core

546 eukaryotic gene set (Simão et al., 2015), (ii) the UniProtKB/Swiss-Prot database, and (iii)

547 the $\mathrm{nr}$ database, using BLASTp and an e-value of 1e-25.

548 To further explore the composition and structure of the CCs, we computed the

549 Pielou equitability index (Mulder et al., 2004), classically used in ecology in order to

550 estimate the richness and/or evenness of species in a sample. Here the Pielou index was

551 used to estimate the contribution of each proteome in a CC, and more precisely for

552 assessing whether a CC is mainly composed of domains from a limited number of

553 proteomes. The index ranges from 0 to 1 , and a high index corresponds to an

554 homogeneous contribution of the proteomes.

555 Investigating functional traits for dinoflagellates

556 Analyses of functional traits were based on the SSN encompassing the 46 557 proteomes derived from "high quality" transcriptomes. The information about 10 selected 558 functional traits was retrieved from the literature (Tab. 1). The details about plastid origin 559 and presence were retrieved from (Caruana and Malin, 2014). Dinoflagellates that are 560 capable of mixotrophy were listed in (Jeong et al., 2010). The information on species with 561 a human (AZP, DSP, NSP, PSP, CFP syndromes) or to marine fauna (ichyotoxicity) 562 toxicity potential was obtained from the Taxonomic Reference List of Harmful MicroAlgae 563 of the IOC-UNESCO (http://www.marinespecies.org/hab/index.php). Dinoflagellate

564 plastidy is reviewed in (Gagat et al., 2014). Dinoflagellates which have the capacity to 565 produce DMSP in high cellular concentration were described in (Caruana et al., 2012).

566 Presence of the theca, characteristic of thecate dinoflagellates, has been studied in (Lin, 567 2011; Orr et al., 2012). In (Rengefors et al., 1998) authors studied dinoflagellates species 
568 that go through a cyst stage during their life cycle. Symbiotic taxa are characterized in

569 (Decelle et al., 2012; Probert et al., 2014; Siano et al., 2010; Trench and Blank, 1987;

570 Yuasa et al., 2016). We later focused on CCs that are specific to a given trait, called "trait-

571 CCs", defined by CCs exclusively composed of vertices tagged with this single trait (and

572 excluding $<\mathrm{NA}>$ tags).

573 Following an exploratory approach, among trait-CCs, CCs including a maximum of

574 distinct proteomes were sought (except for the "parasite" trait, as only one parasite

575 proteomes is represented in the network). In this study, we examined more specifically the

576 functional composition for the "toxicity potential" and "symbiosis" trait-CCs. To validate the

577 SSN capacity to detect trait-CCs characteristic for a given function, we followed a

578 knowledge-based approach searching for sequence similarities through BLASTp (e-value

579 1e-3) to well-known genes from the literature.

580 Focus on the "toxicity potential" functional trait

581 Specific studies on toxic dinoflagellate species have led to the establishment of

582 defined gene sets likely related to toxin production (Snyder et al. 2003; Monroe \& Van

583 Dolah 2008; Wang 2008; Sheng et al. 2010; Kellmann et al. 2010; Stüken et al. 2011;

584 Salcedo et al. 2012; Hackett et al. 2013; Cusick \& Sayler 2013; Lehnert et al. 2014; Perini

585 et al. 2014; Zhang et al. 2014; Kohli et al. 2015, 2016; Meyer et al. 2015; Murray et al.

586 2015; Beedessee et al. 2015). PKS genes are present in all dinoflagellates (Kohli et al.,

587 2015) but many of the toxic metabolites produced by some dinoflagellate species are of

588 polyketide origin (Kellmann et al., 2010). 2,632 polyketide synthase (PKS) peptide

589 sequences from (Kohli et al., 2016) (supplementary data 3) were compared to sequences

590 from "toxicity potential" trait-CCs as well as non-"toxicity potential" trait-CCs as a control

591 (retained alignments show $80 \%$ sequence identity and $80 \%$ sequence coverage).

592 Previous studies have also identified sxtA4 and sxtG genes as related with the STX

593 biosynthesis pathway (Orr et al., 2013). Our investigations in the "toxicity potential" and 
594 non-"toxicity potential" trait-CCs (retained alignments with $80 \%$ sequence identity and $90 \%$

595 sequence coverage) on were based on 26 sxtA4 and 20 sxtG sequences from (Murray et

596 al., 2015) (Tab. S17). The differential composition of functional annotations between

597 "toxicity potential" and non-"toxicity potential" trait-CCs was investigated to detect

598 functions that are likely more represented in toxic species. The counts of each annotation

599 found in each functional category were respectively normalized by the total number of

600 sequences that composed both trait-CCs. Finally, the difference of pair normalized counts

601 for the same annotation in "toxicity potential" and non-"toxicity potential" trait-CCs was

602 calculated (Fig. 2B).

\section{Focus on "symbiosis" functional trait}

604 In this study, three additional transcriptomes of symbiotic species were added to 605 the MMETSP data to increase the number of transcriptomes of symbiotic species from 9 606 to 12. Following a similar strategy as for the "toxicity potential" functional trait, investigation

607 of the "symbiosis" trait in our network was based on reported sets of genes potentially 608 involved in the symbiotic lifestyle for Symbiodinium kawagutii (Lin et al., 2015) and coral 609 symbiotic relationships (Tab. S18). We combined this set with other putative proteins

610 highly up-regulated in anemone-dinoflagellate symbiosis (Lehnert et al., 2014). The

611 distribution of 150 "symbiotic" marker sequences was studied across "symbiosis" trait-CCs

612 (Tab. S12). The differential composition of functional annotations between "symbiosis" and 613 non-"symbiosis" trait-CCs was investigated as previously described for "toxicity potential" 614 trait-CCs.

615

\section{DATA ACCESSIBILITY}

617 SRA numbers for raw files of Brandtodinium nutricula (RCC3468): ERP106907,

618 Gymnoxanthella radiolariae (RCC3507): available soon, Pelagodinium beii (RCC1491): 
619 ERP106909, Heterocapsa sp. (RCC1516): ERP106906 are available on NCBI SRA

620 database.

621 Personal R scripts, SSN file and attribute files for vertices and edges, Fasta files for each

622 assembly of the MMETSP datasets and the corresponding functional annotations, Fasta

623 files and CCs structure files corresponding to trait-CCs for each functional trait, as well

624 as most advanced and extra analyses can be found on figshare:

625 https://figshare.com/projects/Dinoflagellate_SSN/28410

626

\section{Acknowledgments}

628 The authors thank Gaëlle Lelandais, Laure Guillou and Éric Pelletier for their support and

629 critical discussions. The authors We are also grateful to the RCC staff for providing 630 dinoflagellate cultures as well as the Roscoff Bioinformatic platform ABiMS

631 (http://abims.sb-roscoff.fr) for providing computational resources. The authors thank the

632 three anonymous reviewers for their valuable comments and suggestions to improve the

633 quality of the paper. This work was supported by a 3-year Ph.D. grant from "Interface Pour

634 le Vivant" (IPV) program at the Université Pierre et Marie Curie (UPMC), Paris, and this 635 project was supported by grants from Région lle-de-France. 


\section{REFERENCES}

Altschul, S.F., Gish, W., Miller, W., Myers, E.W., and Lipman, D.J. (1990). Basic local alignment search tool. J. Mol. Biol. 215, 403-410.

Alvarez-Ponce, D., Lopez, P., Bapteste, E., and Mclnerney, J.O. (2013). Gene similarity networks provide tools for understanding eukaryote origins and evolution. Proc. Natl. Acad. Sci. U. S. A. 110, E1594-1603.

Aranda, M., Li, Y., Liew, Y.J., Baumgarten, S., Simakov, O., Wilson, M.C., Piel, J., Ashoor, H., Bougouffa, S., Bajic, V.B., et al. (2016). Genomes of coral dinoflagellate symbionts highlight evolutionary adaptations conducive to a symbiotic lifestyle. Sci. Rep. 6.

Armengaud, J., Trapp, J., Pible, O., Geffard, O., Chaumot, A., and Hartmann, E.M. (2014). Non-model organisms, a species endangered by proteogenomics. J. Proteomics 105, 5-18.

Atkinson, H.J., Morris, J.H., Ferrin, T.E., and Babbitt, P.C. (2009). Using Sequence Similarity Networks for Visualization of Relationships Across Diverse Protein Superfamilies. PLoS ONE 4.

Bachvaroff, T.R., Gornik, S.G., Concepcion, G.T., Waller, R.F., Mendez, G.S., Lippmeier, J.C., and Delwiche, C.F. (2014). Dinoflagellate phylogeny revisited: Using ribosomal proteins to resolve deep branching dinoflagellate clades. Mol. Phylogenet. Evol. 70, 314-322.

Beedessee, G., Hisata, K., Roy, M.C., Satoh, N., and Shoguchi, E. (2015). Multifunctional polyketide synthase genes identified by genomic survey of the symbiotic dinoflagellate, Symbiodinium minutum. BMC Genomics 16.

Bittner, L., Halary, S., Payri, C., Cruaud, C., de Reviers, B., Lopez, P., and Bapteste, E. (2010). Some considerations for analyzing biodiversity using integrative metagenomics and gene networks. Biol. Direct 5, 47.

Bolger, A.M., Lohse, M., and Usadel, B. (2014). Trimmomatic: A flexible trimmer for Illumina Sequence Data. Bioinformatics btu170.

Brocchieri, L., and Karlin, S. (2005). Protein length in eukaryotic and prokaryotic proteomes. Nucleic Acids Res. 33, 3390-3400.

Buchfink, B., Xie, C., and Huson, D.H. (2015). Fast and sensitive protein alignment using DIAMOND. Nat. Methods 12, 59-60.

Caron, D.A., Alexander, H., Allen, A.E., Archibald, J.M., Armbrust, E.V., Bachy, C., Bell, C.J., Bharti, A., Dyhrman, S.T., Guida, S.M., et al. (2016). Probing the evolution, ecology and physiology of marine protists using transcriptomics. Nat. Rev. Microbiol. advance online publication. 
Caruana, A.M.N., and Malin, G. (2014). The variability in DMSP content and DMSP lyase activity in marine dinoflagellates. Prog. Oceanogr. 120, 410-424.

Caruana, A.M.N., Steinke, M., Turner, S.M., and Malin, G. (2012). Concentrations of dimethylsulphoniopropionate and activities of dimethylsulphide-producing enzymes in batch cultures of nine dinoflagellate species. Biogeochemistry 110, 87-107.

Charpentier, M., Bredemeier, R., Wanner, G., Takeda, N., Schleiff, E., and Parniske, M. (2008). Lotus japonicus CASTOR and POLLUX Are lon Channels Essential for Perinuclear Calcium Spiking in Legume Root Endosymbiosis. Plant Cell 20, 3467-3479.

Cheng, S., Karkar, S., Bapteste, E., Yee, N., Falkowski, P., and Bhattacharya, D. (2014). Sequence similarity network reveals the imprints of major diversification events in the evolution of microbial life. Front. Ecol. Evol. 2.

Csárdi, G., and Nepusz, T. (2006). The igraph software package for complex network research. InterJournal Complex Syst.

Cusick, K.D., and Sayler, G.S. (2013). An Overview on the Marine Neurotoxin, Saxitoxin: Genetics, Molecular Targets, Methods of Detection and Ecological Functions. Mar. Drugs 11, 991-1018.

Day, E.K., Sosale, N.G., and Lazzara, M.J. (2016). Cell signaling regulation by protein phosphorylation: a multivariate, heterogeneous, and context-dependent process. Curr. Opin. Biotechnol. 40, 185-192.

Decelle, J., Probert, I., Bittner, L., Desdevises, Y., Colin, S., Vargas, C. de, Galí, M., Simó, R., and Not, F. (2012). An original mode of symbiosis in open ocean plankton. Proc. Natl. Acad. Sci. 109, 18000-18005.

Decelle, J., Colin, S., and Foster, R.A. (2015). Photosymbiosis in Marine Planktonic Protists. In Marine Protists, S. Ohtsuka, T. Suzaki, T. Horiguchi, N. Suzuki, and F. Not, eds. (Springer Japan), pp. 465-500.

Dorrell, R.G., Gile, G., McCallum, G., Méheust, R., Bapteste, E.P., Klinger, C.M., Brillet-Guéguen, L., Freeman, K.D., Richter, D.J., and Bowler, C. (2017). Chimeric origins of ochrophytes and haptophytes revealed through an ancient plastid proteome. ELife 6, e23717.

Dupont, C.L., McCrow, J.P., Valas, R., Moustafa, A., Walworth, N., Goodenough, U., Roth, R., Hogle, S.L., Bai, J., Johnson, Z.I., et al. (2015). Genomes and gene expression across light and productivity gradients in eastern subtropical Pacific microbial communities. ISME J. 9, 1076-1092. 
Flewelling, L.J., Naar, J.P., Abbott, J.P., Baden, D.G., Barros, N.B., Bossart, G.D., Bottein, M.-Y.D., Hammond, D.G., Haubold, E.M., Heil, C.A., et al. (2005). Brevetoxicosis: Red tides and marine mammal mortalities. Nature 435, 755-756.

Forster, D., Bittner, L., Karkar, S., Dunthorn, M., Romac, S., Audic, S., Lopez, P., Stoeck, T., and Bapteste, E. (2015). Testing ecological theories with sequence similarity networks: marine ciliates exhibit similar geographic dispersal patterns as multicellular organisms. BMC Biol. 13, 16.

Gagat, P., Bodył, A., Mackiewicz, P., and Stiller, J.W. (2014). Tertiary Plastid Endosymbioses in Dinoflagellates. In Endosymbiosis, W. Löffelhardt, ed. (Springer Vienna), pp. 233-290.

Gast, R.J., Moran, D.M., Dennett, M.R., and Caron, D.A. (2007). Kleptoplasty in an Antarctic dinoflagellate: caught in evolutionary transition? Environ. Microbiol. 9, 39-45.

Gerlt, J.A., Babbitt, P.C., Jacobson, M.P., and Almo, S.C. (2012). Divergent Evolution in Enolase Superfamily: Strategies for Assigning Functions. J. Biol. Chem. 287, 29-34.

Germot, A., and Philippe, H. (1999). Critical Analysis of Eukaryotic Phylogeny: A Case Study Based on the HSP70 Family. J. Eukaryot. Microbiol. 46, 116-124.

Goodson, M.S., Whitehead, L.F., and Douglas, A.E. (2001). Symbiotic dinoflagellates in marine Cnidaria: diversity and function. Hydrobiologia 461, 7982.

Grabherr, M.G., Haas, B.J., Yassour, M., Levin, J.Z., Thompson, D.A., Amit, I., Adiconis, X., Fan, L., Raychowdhury, R., Zeng, Q., et al. (2011). Full-length transcriptome assembly from RNA-Seq data without a reference genome (Trinity). Nat. Biotechnol. 29, 644-652.

Guiry, M.D., and Guiry, G.M. (2018). AlgaeBase.

Haas, B.J., Papanicolaou, A., Yassour, M., Grabherr, M., Blood, P.D., Bowden, J., Couger, M.B., Eccles, D., Li, B., Lieber, M., et al. (2013). De novo transcript sequence reconstruction from RNA-seq using the Trinity platform for reference generation and analysis. Nat. Protoc. 8, 1494-1512.

Hackett, J.D., Wisecaver, J.H., Brosnahan, M.L., Kulis, D.M., Anderson, D.M., Bhattacharya, D., Plumley, F.G., and Erdner, D.L. (2013). Evolution of Saxitoxin Synthesis in Cyanobacteria and Dinoflagellates. Mol. Biol. Evol. 30, 70-78.

Jaeckisch, N., Yang, I., Wohlrab, S., Glöckner, G., Kroymann, J., Vogel, H., Cembella, A., and John, U. (2011). Comparative Genomic and Transcriptomic Characterization of the Toxigenic Marine Dinoflagellate Alexandrium ostenfeldii. PLOS ONE 6, e28012. 
Janouškovec, J., Gavelis, G.S., Burki, F., Dinh, D., Bachvaroff, T.R., Gornik, S.G., Bright, K.J., Imanian, B., Strom, S.L., Delwiche, C.F., et al. (2016). Major transitions in dinoflagellate evolution unveiled by phylotranscriptomics. Proc. Natl. Acad. Sci. 201614842.

Jeong, H.J., Yoo, Y.D., Kim, J.S., Seong, K.A., Kang, N.S., and Kim, T.H. (2010). Growth, feeding and ecological roles of the mixotrophic and heterotrophic dinoflagellates in marine planktonic food webs. Ocean Sci. J. 45, 65-91.

Jones, P., Binns, D., Chang, H.-Y., Fraser, M., Li, W., McAnulla, C., McWilliam, H., Maslen, J., Mitchell, A., Nuka, G., et al. (2014). InterProScan 5: genome-scale protein function classification. Bioinforma. Oxf. Engl. 30, 1236-1240.

Keeling, P.J., Burki, F., Wilcox, H.M., Allam, B., Allen, E.E., Amaral-Zettler, L.A., Armbrust, E.V., Archibald, J.M., Bharti, A.K., Bell, C.J., et al. (2014). The Marine Microbial Eukaryote Transcriptome Sequencing Project (MMETSP): Illuminating the Functional Diversity of Eukaryotic Life in the Oceans through Transcriptome Sequencing. PLOS Biol 12, e1001889.

Keller, M.B., Lavori, P.W., Friedman, B., Nielsen, E., Endicott, J., McDonaldScott, P., and Andreasen, N.C. (1987). The Longitudinal Interval Follow-up Evaluation. A comprehensive method for assessing outcome in prospective longitudinal studies. Arch. Gen. Psychiatry 44, 540-548.

Kellmann, R., Stüken, A., Orr, R.J.S., Svendsen, H.M., and Jakobsen, K.S. (2010). Biosynthesis and Molecular Genetics of Polyketides in Marine Dinoflagellates. Mar. Drugs 8, 1011-1048.

Khosla, C., Herschlag, D., Cane, D.E., and Walsh, C.T. (2014). Assembly Line Polyketide Synthases: Mechanistic Insights and Unsolved Problems.

Biochemistry (Mosc.) 53, 2875-2883.

Kohli, G.S., John, U., Figueroa, R.I., Rhodes, L.L., Harwood, D.T., Groth, M., Bolch, C.J.S., and Murray, S.A. (2015). Polyketide synthesis genes associated with toxin production in two species of Gambierdiscus (Dinophyceae). BMC Genomics 16, 410.

Kohli, G.S., John, U., Van Dolah, F.M., and Murray, S.A. (2016). Evolutionary distinctiveness of fatty acid and polyketide synthesis in eukaryotes. ISME J.

Langmead, B., Trapnell, C., Pop, M., and Salzberg, S.L. (2009). Ultrafast and memory-efficient alignment of short DNA sequences to the human genome. Genome Biol. 10, R25.

Le Bescot, N., Mahé, F., Audic, S., Dimier, C., Garet, M.-J., Poulain, J., Wincker, P., de Vargas, C., and Siano, R. (2016). Global patterns of pelagic dinoflagellate diversity across protist size classes unveiled by metabarcoding. Environ. Microbiol. 18, 609-626. 
Lee, R., Lai, H., Malik, S.B., Saldarriaga, J.F., Keeling, P.J., and Slamovits, C.H. (2014). Analysis of EST data of the marine protist Oxyrrhis marina, an emerging model for alveolate biology and evolution. BMC Genomics 15, 122.

Lehnert, E.M., Mouchka, M.E., Burriesci, M.S., Gallo, N.D., Schwarz, J.A., and Pringle, J.R. (2014). Extensive Differences in Gene Expression Between Symbiotic and Aposymbiotic Cnidarians. G3 GenesGenomesGenetics 4, $277-$ 295.

Lima-Mendez, G., Faust, K., Henry, N., Decelle, J., Colin, S., Carcillo, F., Chaffron, S., Ignacio-Espinosa, J.C., Roux, S., Vincent, F., et al. (2015).

Determinants of community structure in the global plankton interactome. Science 348, 1262073.

Lin, S. (2011). Genomic understanding of dinoflagellates. Res. Microbiol. 162, 551-569.

Lin, S., Cheng, S., Song, B., Zhong, X., Lin, X., Li, W., Li, L., Zhang, Y., Zhang, $\mathrm{H}$., Ji, Z., et al. (2015). The Symbiodinium kawagutii genome illuminates dinoflagellate gene expression and coral symbiosis. Science 350, 691-694.

Lionetti, V., and Metraux, J.-P. (2015). Plant cell wall in pathogenesis, parasitism and symbiosis (Frontiers Media SA).

Lopez, P., Halary, S., and Bapteste, E. (2015). Highly divergent ancient gene families in metagenomic samples are compatible with additional divisions of life. Biol. Direct 10, 64.

Marchler-Bauer, A., Anderson, J.B., Cherukuri, P.F., DeWeese-Scott, C., Geer, L.Y., Gwadz, M., He, S., Hurwitz, D.I., Jackson, J.D., Ke, Z., et al. (2005). CDD: a Conserved Domain Database for protein classification. Nucleic Acids Res. 33, D192-D196.

Massana, R., Gobet, A., Audic, S., Bass, D., Bittner, L., Boutte, C., Chambouvet, A., Christen, R., Claverie, J.-M., Decelle, J., et al. (2015). Marine protist diversity in European coastal waters and sediments as revealed by high-throughput sequencing. Environ. Microbiol. 17, 4035-4049.

Matzke, M., Weiger, T.M., Papp, I., and Matzke, A.J.M. (2009). Nuclear membrane ion channels mediate root nodule development. Trends Plant Sci. 14, 295-298.

Méheust, R., Zelzion, E., Bhattacharya, D., Lopez, P., and Bapteste, E. (2016). Protein networks identify novel symbiogenetic genes resulting from plastid endosymbiosis. Proc. Natl. Acad. Sci. 113, 3579-3584.

Meyer, J.M., Rödelsperger, C., Eichholz, K., Tillmann, U., Cembella, A., McGaughran, A., and John, U. (2015). Transcriptomic characterisation and 
genomic glimps into the toxigenic dinoflagellate Azadinium spinosum, with emphasis on polykeitde synthase genes. BMC Genomics 16.

Monroe, E.A., and Van Dolah, F.M. (2008). The Toxic Dinoflagellate Karenia brevis Encodes Novel Type I-like Polyketide Synthases Containing Discrete Catalytic Domains. Protist 159, 471-482.

Montagnes, D.J.S., Lowe, C.D., Roberts, E.C., Breckels, M.N., Boakes, D.E., Davidson, K., Keeling, P.J., Slamovits, C.H., Steinke, M., Yang, Z., et al. (2011). An introduction to the special issue: Oxyrrhis marina, a model organism? J. Plankton Res. 33, 549-554.

Mulder, C.P.H., Bazeley-White, E., Dimitrakopoulos, P.G., Hector, A., SchererLorenzen, M., and Schmid, B. (2004). Species evenness and productivity in experimental plant communities. Oikos 107, 50-63.

Murray, S.A., Diwan, R., Orr, R.J.S., Kohli, G.S., and John, U. (2015). Gene duplication, loss and selection in the evolution of saxitoxin biosynthesis in alveolates. Mol. Phylogenet. Evol. 92, 165-180.

Murray, S.A., Suggett, D.J., Doblin, M.A., Kohli, G.S., Seymour, J.R., Fabris, M., and Ralph, P.J. (2016). Unravelling the functional genetics of dinoflagellates: a review of approaches and opportunities. Perspect. Phycol. 37-52.

Orr, R.J.S., Murray, S.A., Stüken, A., Rhodes, L., and Jakobsen, K.S. (2012). When Naked Became Armored: An Eight-Gene Phylogeny Reveals Monophyletic Origin of Theca in Dinoflagellates. PLoS ONE 7, e50004.

Orr, R.J.S., Stüken, A., Murray, S.A., and Jakobsen, K.S. (2013). Evolutionary Acquisition and Loss of Saxitoxin Biosynthesis in Dinoflagellates: the Second "Core" Gene, sxtG. Appl. Environ. Microbiol. 79, 2128-2136.

Parra, G., Bradnam, K., and Korf, I. (2007). CEGMA: a pipeline to accurately annotate core genes in eukaryotic genomes. Bioinforma. Oxf. Engl. 23, 10611067.

Perini, F., Galluzzi, L., Dell'Aversano, C., Dello lacovo, E., Tartaglione, L., Ricci, F., Forino, M., Ciminiello, P., and Penna, A. (2014). SxtA and sxtG Gene Expression and Toxin Production in the Mediterranean Alexandrium minutum (Dinophyceae). Mar. Drugs 12, 5258-5276.

Probert, I., Siano, R., Poirier, C., Decelle, J., Biard, T., Tuji, A., Suzuki, N., and Not, F. (2014). Brandtodinium gen. nov. and B. nutricula comb. Nov. (Dinophyceae), a dinoflagellate commonly found in symbiosis with polycystine radiolarians. J. Phycol. 50, 388-399.

Rengefors, K., Karlsson, I., and Hansson, L.-A. (1998). Algal cyst dormancy: a temporal escape from herbivory. Proc. R. Soc. B Biol. Sci. 265, 1353-1358. 
Salcedo, T., Upadhyay, R.J., Nagasaki, K., and Bhattacharya, D. (2012). Dozens of Toxin-Related Genes Are Expressed in a Nontoxic Strain of the Dinoflagellate Heterocapsa circularisquama. Mol. Biol. Evol. 29, 1503-1506.

Sheng, J., Malkiel, E., Katz, J., Adolf, J.E., and Place, A.R. (2010). A dinoflagellate exploits toxins to immobilize prey prior to ingestion. Proc. Natl. Acad. Sci. 107, 2082-2087.

Shoguchi, E., Shinzato, C., Kawashima, T., Gyoja, F., Mungpakdee, S., Koyanagi, R., Takeuchi, T., Hisata, K., Tanaka, M., Fujiwara, M., et al. (2013). Draft Assembly of the Symbiodinium minutum Nuclear Genome Reveals Dinoflagellate Gene Structure. Curr. Biol. 23, 1399-1408.

Siano, R., Montresor, M., Probert, I., Not, F., and de Vargas, C. (2010). Pelagodinium gen. nov. and P. béii comb. nov., a dinoflagellate symbiont of planktonic foraminifera. Protist 161, 385-399.

Siano, R., Alves-de-Souza, C., Foulon, E., Bendif, E.M., Simon, N., Guillou, L., and Not, F. (2011). Distribution and host diversity of Amoebophryidae parasites across oligotrophic waters of the Mediterranean Sea. Biogeosciences 8, 267278.

Sibbald, S.J., and Archibald, J.M. (2017). More protist genomes needed. Nat. Ecol. Evol. 1, 0145.

Simão, F.A., Waterhouse, R.M., loannidis, P., Kriventseva, E.V., and Zdobnov, E.M. (2015). BUSCO: assessing genome assembly and annotation completeness with single-copy orthologs. Bioinformatics btv351.

Snyder, R.V., Gibbs, P.D.L., Palacios, A., Abiy, L., Dickey, R., Lopez, J.V., and Rein, K.S. Polyketide Synthase Genes from Marine Dinoflagellates. Mar. Biotechnol. 5, 1-12.

Stoecker, D.K., Hansen, P.J., Caron, D.A., and Mitra, A. (2017). Mixotrophy in the Marine Plankton. Annu. Rev. Mar. Sci. 9, 311-335.

Stüken, A., Orr, R.J.S., Kellmann, R., Murray, S.A., Neilan, B.A., and Jakobsen, K.S. (2011). Discovery of Nuclear-Encoded Genes for the Neurotoxin Saxitoxin in Dinoflagellates. PLOS ONE 6, e20096.

Trench, R.K., and Blank, R.J. (1987). Symbiodinium Microadriaticum Freudenthal, S. Goreauii Sp. Nov., S. Kawagutii Sp. Nov. and S. Pilosum Sp. Nov.: Gymnodinioid Dinoflagellate Symbionts of Marine Invertebrates 1. J. Phycol. 23, 469-481.

Vonk, F.J., Casewell, N.R., Henkel, C.V., Heimberg, A.M., Jansen, H.J., McCleary, R.J.R., Kerkkamp, H.M.E., Vos, R.A., Guerreiro, I., Calvete, J.J., et al. 
(2013). The king cobra genome reveals dynamic gene evolution and adaptation in the snake venom system. Proc. Natl. Acad. Sci. 110, 20651-20656.

Wang, D.-Z. (2008). Neurotoxins from Marine Dinoflagellates: A Brief Review. Mar. Drugs 6, 349-371.

Yang, Y., and Smith, S.A. (2013). Optimizing de novo assembly of short-read RNA-seq data for phylogenomics. BMC Genomics 14, 328.

Yang, L., Tan, J., O’Brien, E.J., Monk, J.M., Kim, D., Li, H.J., Charusanti, P., Ebrahim, A., Lloyd, C.J., Yurkovich, J.T., et al. (2015). Systems biology definition of the core proteome of metabolism and expression is consistent with highthroughput data. Proc. Natl. Acad. Sci. 112, 10810-10815.

Yuasa, T., Horiguchi, T., Mayama, S., and Takahashi, O. (2016). Gymnoxanthella radiolariae gen. et sp. nov. (Dinophyceae), a dinoflagellate symbiont from solitary polycystine radiolarians. J. Phycol. 52, 89-104.

Zhang, Y., Zhang, S.-F., Lin, L., and Wang, D.-Z. (2014). Comparative Transcriptome Analysis of a Toxin-Producing Dinoflagellate Alexandrium catenella and Its Non-Toxic Mutant. Mar. Drugs 12, 5698-5718. 


\section{Tab 1. Transcriptomes taxonomy, assembly metrics and functional traits}

Summary table of the 46 transcriptomes (and the corresponding strains) analyzed in this study, ranked based on their taxonomy. For 3 datasets, strain has been pooled: Oxyrrhis marina strains (NA and LB1974), the two Prorocentrum minimum strains (CCMP1329 and CCMP2233) and the two Polarella glacialis strains (CCMP1383 and CCMP2088). In contrast we kept separated strains from MMETSP and our datasets: the two Pelagodinium beii RCC1491 strains (see M\&M section). Assembly metrics are reported for each transcriptome encompassing: the number of assembled contigs, N50, the remapping rate of initial reads, the number of predicted protein domains found in transcript sequences and the number of functional annotations identified through Interproscan 5 . The proteomes derived from the 46 transcriptomes presented here are included in the sequence similarity network. Based on a literature survey, information about functional traits for each species included in the dataset is provided: chloroplast type (P: peridinin, $\mathrm{H}$ : haptophyte-like, $\mathrm{C}$ : cryptomonad-like, $\mathrm{D}$ : diatom-like, $\mathrm{R}$ : remnant or absent plastid, NC: non-consitutive chloroplast), mixotrophy, toxicity potential (DSP:Diarrhetic shellfish poisoning, CFP:Ciguatera Fish Poisoning, PSP:Paralytic shellfish poisoning, AZP:Azaspiracid Shellfish Poisoning, NSP:Neurologic Shellfish Poisoning), ability to be symbionts, kleptoplasty, ichyotoxicity, parasitism, ability to produce DSMP, presence of a theca, ability to form cysts during life-cycle. <NA> corresponds to a lack of information.

Guiry, M.D. \& Guiry, G.M. 2018. AlgaeBase. World-wide electronic publication, National University of Ireland, Galway. http://www.algaebase.org; searched on 08 February 2018. 
Transcriptome taxonomy, mertics \& tunction tralts

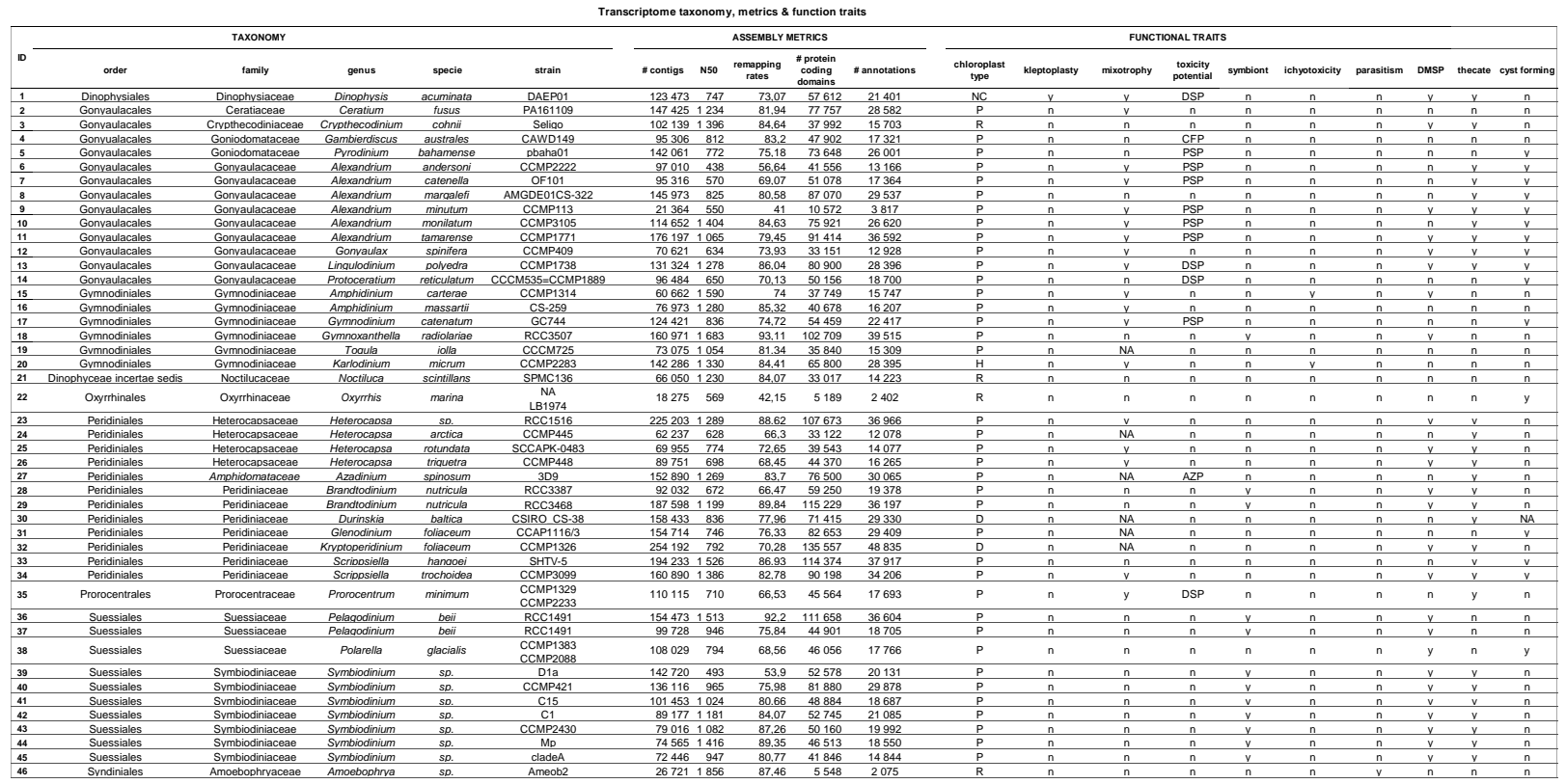


Fig. 1. Unveiling the core, accessory and pan proteome of $\mathbf{4 3}$ dinoflagellates proteomes.

(A) Number of connected components (CCs) in the core (green), accessory (red) and pan (blue) dinoflagellate proteomes, considering 2 to 43 proteomes. (B) Comparison of the 37,842 protein domains included in the 252 core dinoflagellates CCs to BUSCO, UniProtKB/Swiss-Prot and $\mathrm{nr}$ databases. The number and percentage of core sequences with at least one match in each database, and the number and percentage of their corresponding CCs.

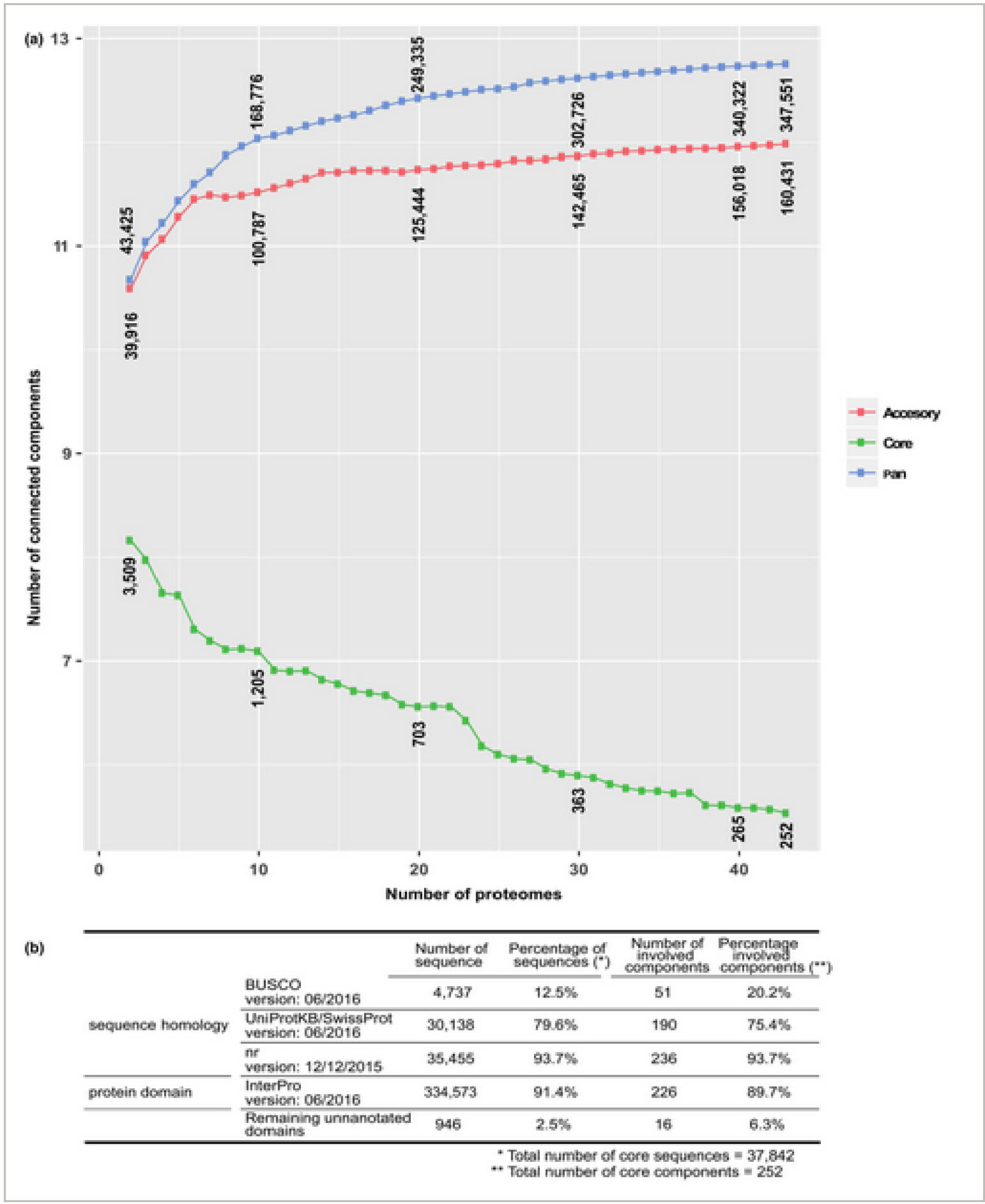


Fig. 2. Exploring functions in toxicity potential trait-CCs and symbiosis trait-CCs.

(A and D) Top 10 functional annotations (GOslim levels) of sequences belonging to the 45,207 "toxicity potential" trait-CCs (A) and to the 90,794 "symbiosis" trait-CCs (D). (B and E) Differential composition of functional annotations between "toxicity potential" and non-"toxicity potential" trait-CCs (B) and "symbiosis" and non"symbiosis" trait-CCs (E). (C and F) The circular barplot shows the number of connected components that include 1 to 14 proteome(s) of the transcriptomes assigned to toxic species (C) and the number of connected components that include 1 to 12 proteome(s) of the transcriptomes assigned to symbiotic species $(F)$.

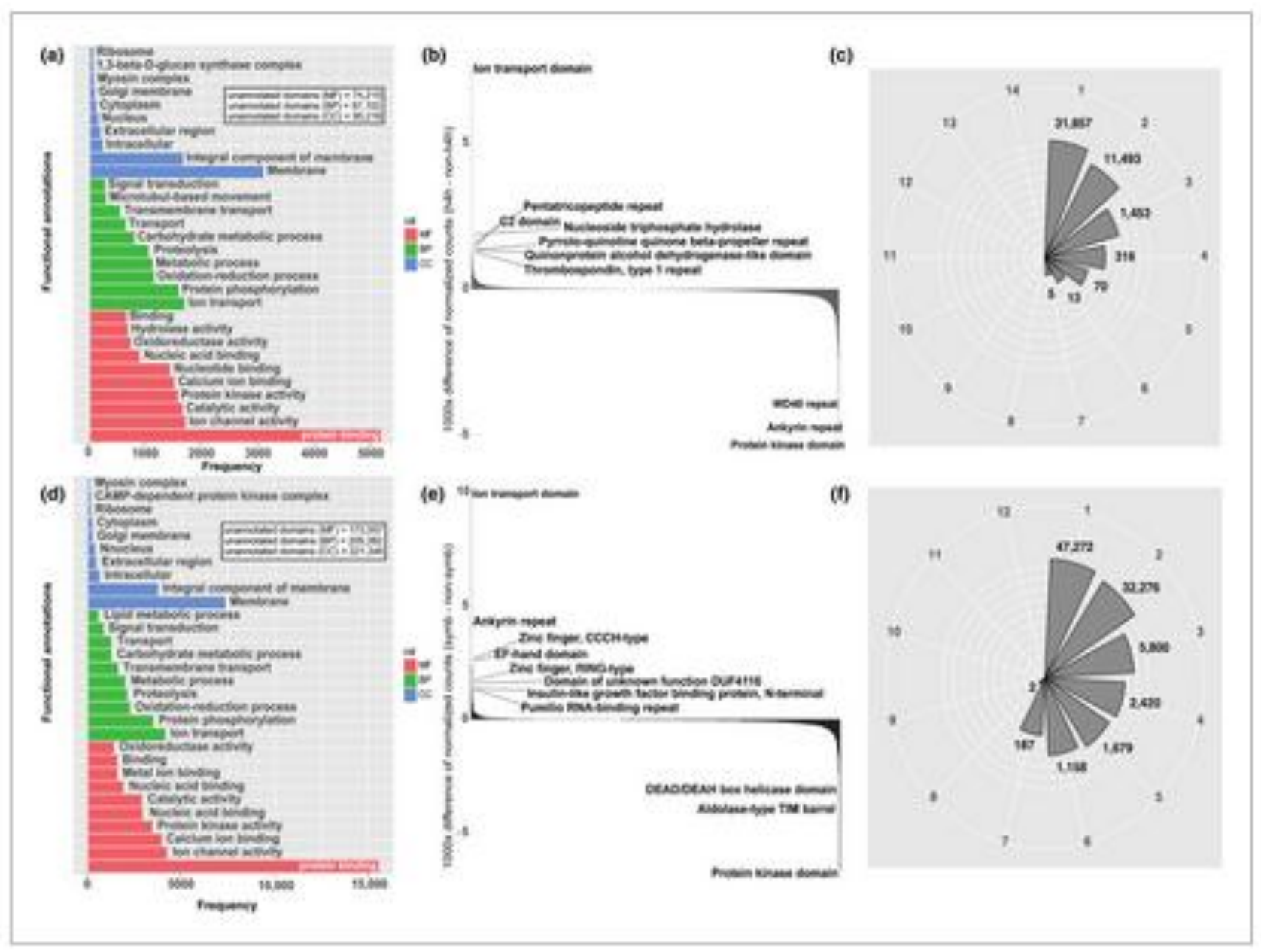


Fig. S1: Optimal sequence identity threshold selection. The cutoff was chosen such that: $(A)$ the network contains a maximum of connected components with homogeneous functional annotation (i.e. a unique GO Slim term for all annotated protein coding domains in each $\mathrm{CC}$ ) and (B) the network conserved a maximum of « large " connected components.

Fig. S2: Top 10 functional annotations (GOslim levels) in all core components. The three levels of annotation are represented: Molecular Function level (MF, red), Biological Process (BP, green) and Cellular Component (CC, blue).

Fig. S3: Overlap between the 101 multiple protein alignments from (2) (used for phylogenomics) and our core dinoflagellate proteome. The number of copies of the 101 proteins is represented here in a heat map: the blue color gradient represents the number of orthologous sequences available of a particular protein ( $y$-axis) for each studied dinoflagellate species in (2) (x-axis). The overlap between the data set from (2) and our core proteome is represented by the red boxes: i.e. each protein sequence from (2) that aligned to at least a core protein coding domain is here delimited in red.

Fig. S4: (A) Number of connected components for each functional trait. (B) Proportion of annotated sequences of connected components for each functional trait.

Fig. S5: Pipeline diagram of our analysis composed of 5 distinct steps (for more details see Material \& Methods): (1) Preprocessing step including read quality evaluation and filtering; (2) De novo assembly step in which assembled contigs were generated from cleaned reads with Trinity (ref. 57). (3) Quality evaluation of the previously assembled contigs. (4) Downstream analysis divided into two parts, with first detection of likely coding domains within contig sequences and then functional annotation of previously detected domains. (5) Construction of a sequence similarity network based on de novo assembly and downstream analysis results.

Fig. S6: A connected component outline. At the top, a multiple alignment of 3 protein coding domain sequences $A, B$ and $C$. The 3 alignments respect sequence identity threshold $(>60 \%)$ and sequence coverage threshold $(>80 \%)$. At the bottom, a sketch of the corresponding connected component where protein coding domain sequences are represented by vertices and each alignment between two sequences is represented by and edge.

Table. S7: Table of all datasets used in this work with MMETSP IDs. Taxonomy, functional traits information and the presence in the SSN has been indicated in the table for each entry.

Fig. S8: Number of peptide sequences per proteome derived from "high quality" transcriptomes. Red line represents the minimum number of sequences threshold ( 9,000 peptide sequences) required to perform core/accessory/pan proteome analysis. 

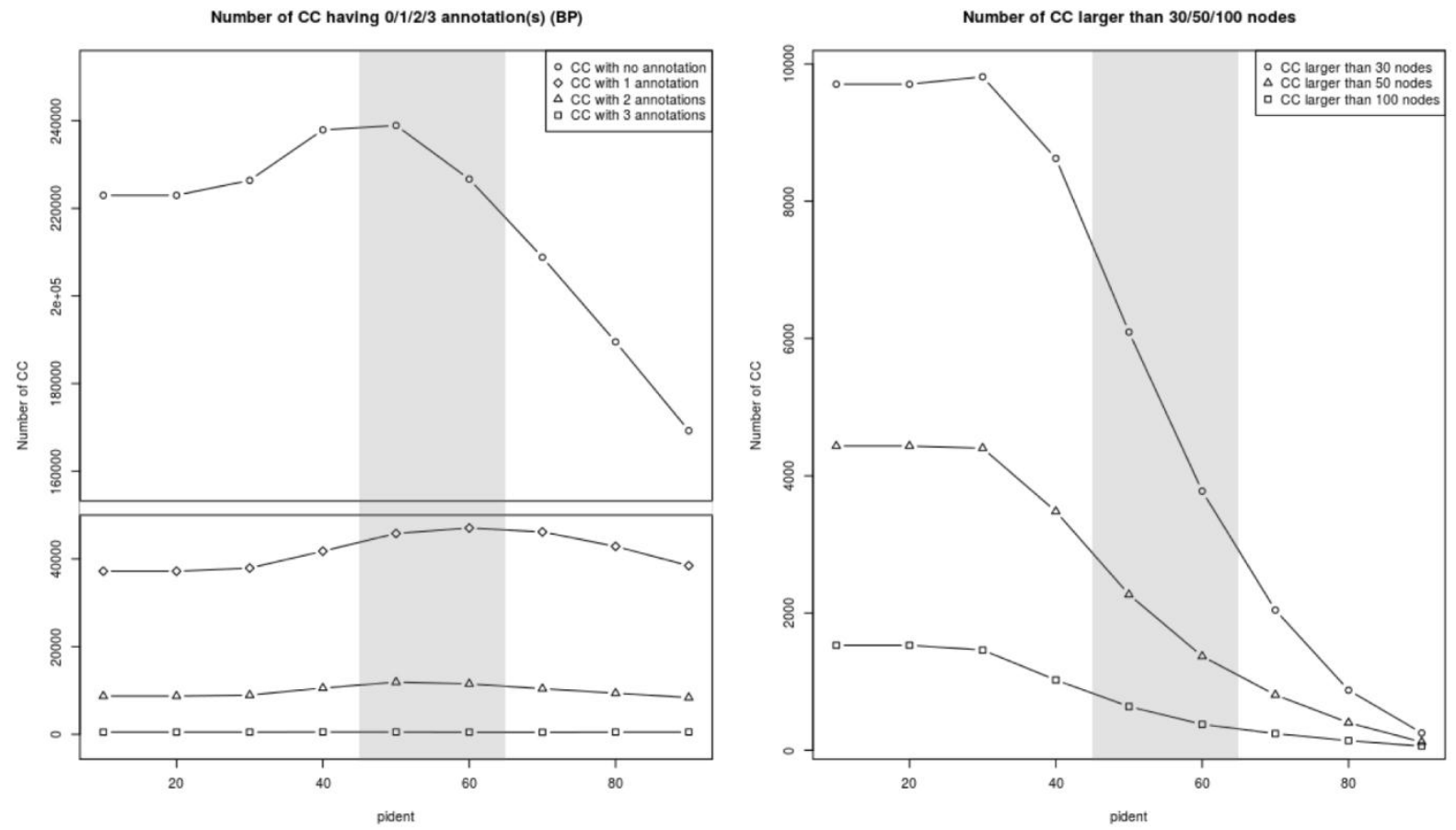

Fig. S 1: Optimal sequence identity threshold selection. The cutoff was chosen such that: $(A)$ the network contains a maximum of connected components with homogeneous functional annotation (i.e. a unique GO Slim term for all annotated protein coding domains in each $\mathrm{CC}$ ) and (B) the network conserved a maximum of «large » connected components. 


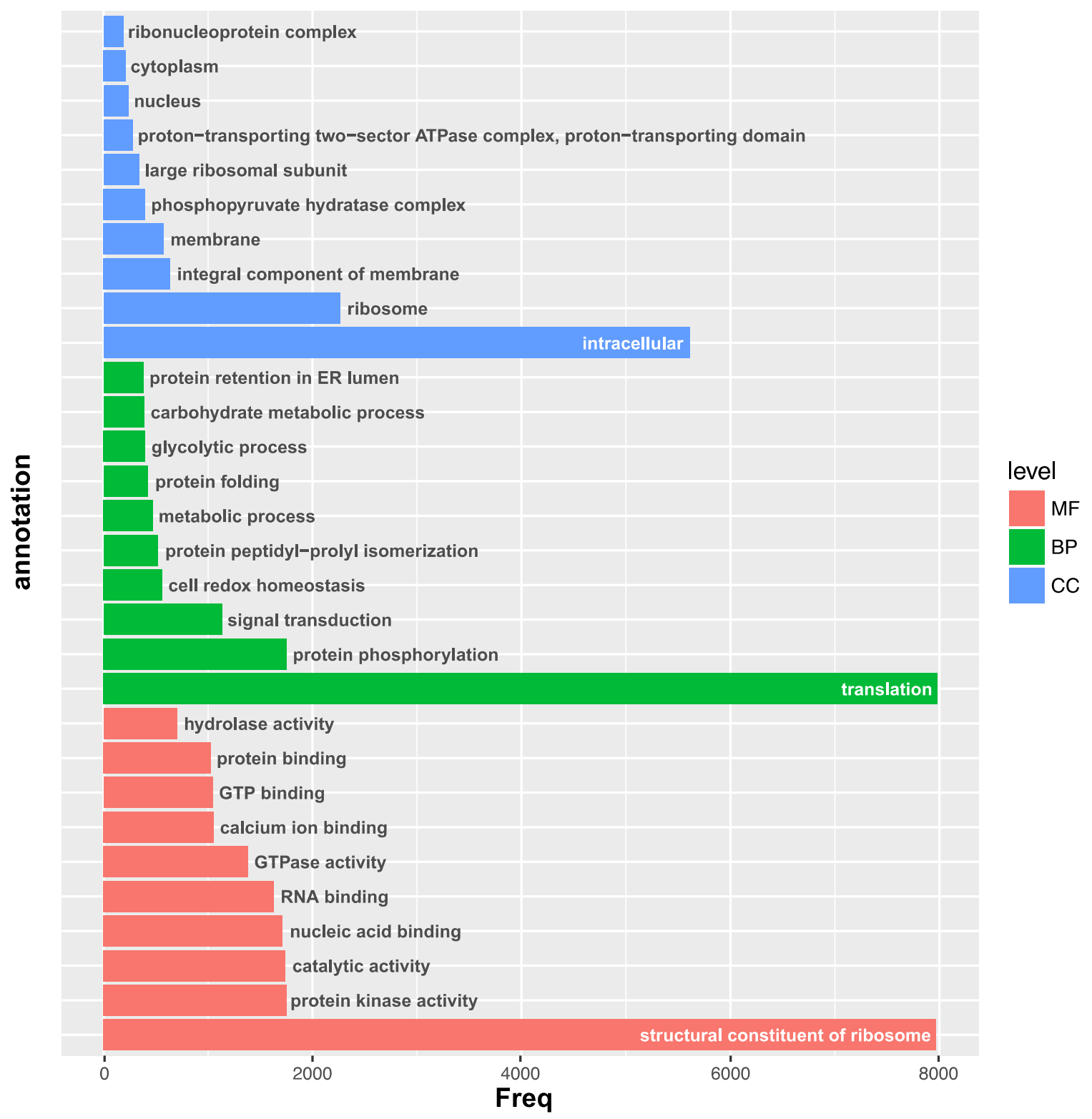

Fig. S2: Top 10 functional annotations (GOslim levels) in all core components. The three levels of annotation are represented: Molecular Function level (MF, red), Biological Process (BP, green) and Cellular Component (CC, blue). 


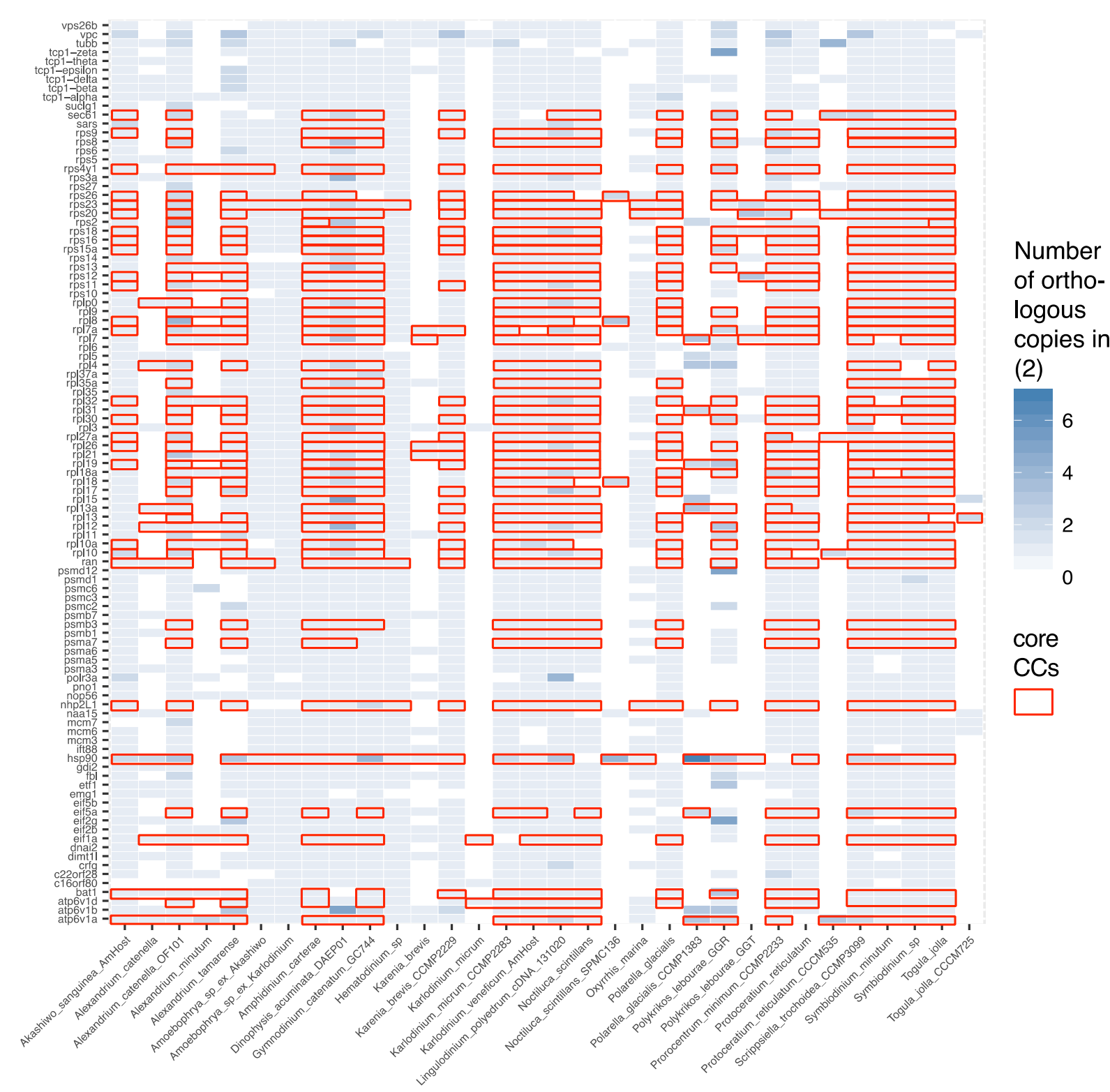

Fig. $S 3$ : Overlap between the 101 multiple protein alignments from (2) (used for phylogenomics) and our core dinoflagellate proteome. The number of copies of the 101 proteins is represented here in a heat map: the blue color gradient represents the number of orthologous sequences available of a particular protein (y-axis) for each studied dinoflagellate species in (2) (x-axis). The overlap between the data set from (2) and our core proteome is represented by the red boxes: i.e. each protein sequence from (2) that aligned to at least a core protein coding domain is here delimited in red. 


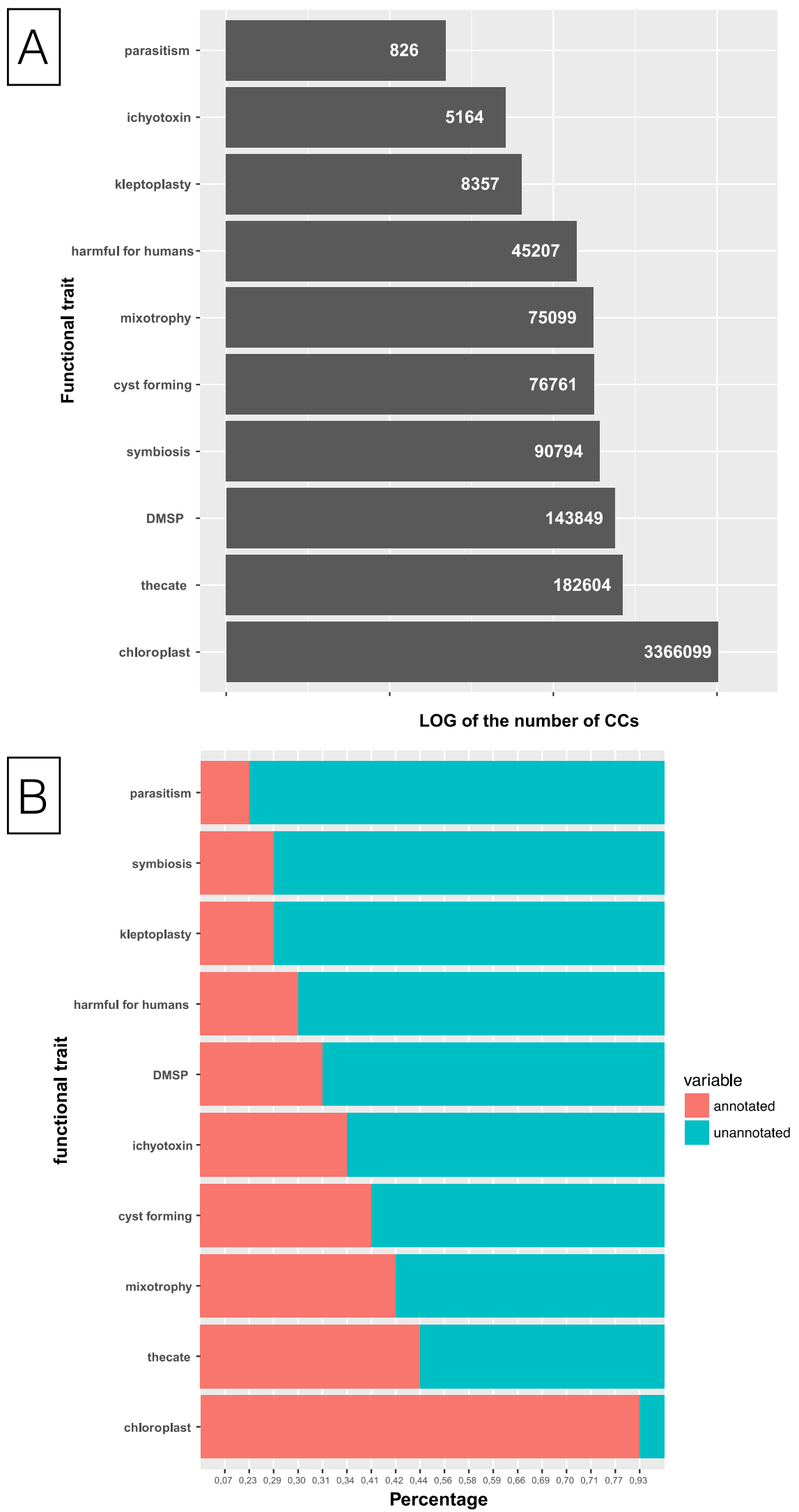

Fig. S 4: (A) Number of connected components for each functional trait. (B) Proportion of annotated sequences of connected components for each functional trait. 


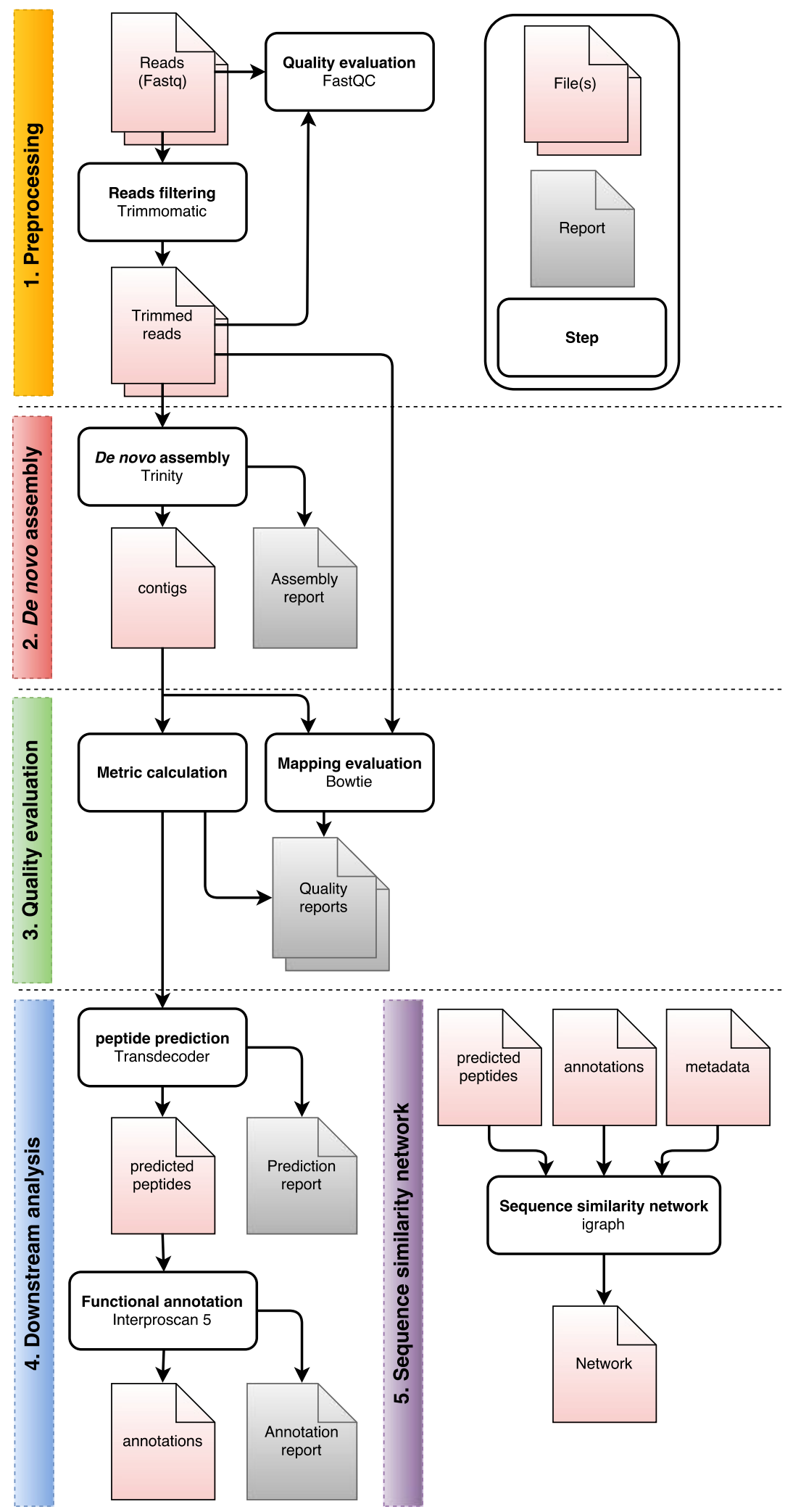

Fig. S 5: Pipeline diagram of our analysis composed of 5 distinct steps (for more details see Material \& Methods): (1) Preprocessing step including read quality evaluation and filtering; (2) De novo assembly step in which assembled contigs were generated from cleaned reads with Trinity (ref. 57). (3) Quality evaluation of the previously assembled contigs. (4) Downstream analysis divided into two parts, with first detection of likely coding domains within contig sequences and then functional annotation of previously detected domains. (5) Construction of a sequence similarity network based on de novo assembly and downstream analysis results. 
protein coding domain A

protein coding domain B

protein coding domain $\mathrm{C}$

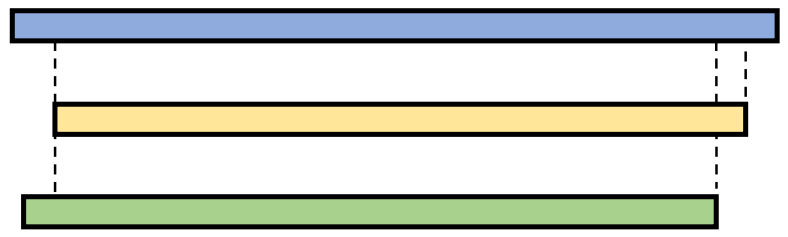

multiple alignement

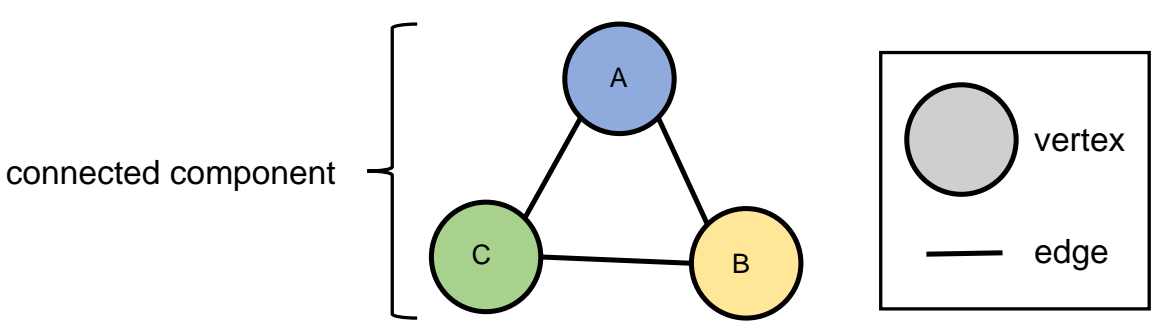

Fig. S 6: A connected component outline. At the top, a multiple alignment of 3 protein coding domain sequences $A, B$ and $C$. The 3 alignments respect sequence identity threshold (>60\%) and sequence coverage threshold (>80\%). At the bottom, a sketch of the corresponding connected component where protein coding domain sequences are represented by vertices and each alignment between two sequences is represented by and edge. 
Fig. 5 7: Table of the 60 datasets used in this study. It encompasses both MMETSP subsets with their ID and the 4 newly added datasets of our contribution ( $n^{\circ} 57-60$ marked with *). We were not able to complete the assembly for $n^{\circ} 22,27$ and 47. We defined low quality transcriptomes which show metrics that do not fit the quality threshold that we defined (see $M \& M): n^{\circ} 8,16,20,26,32,37,41,42,44,56$. 


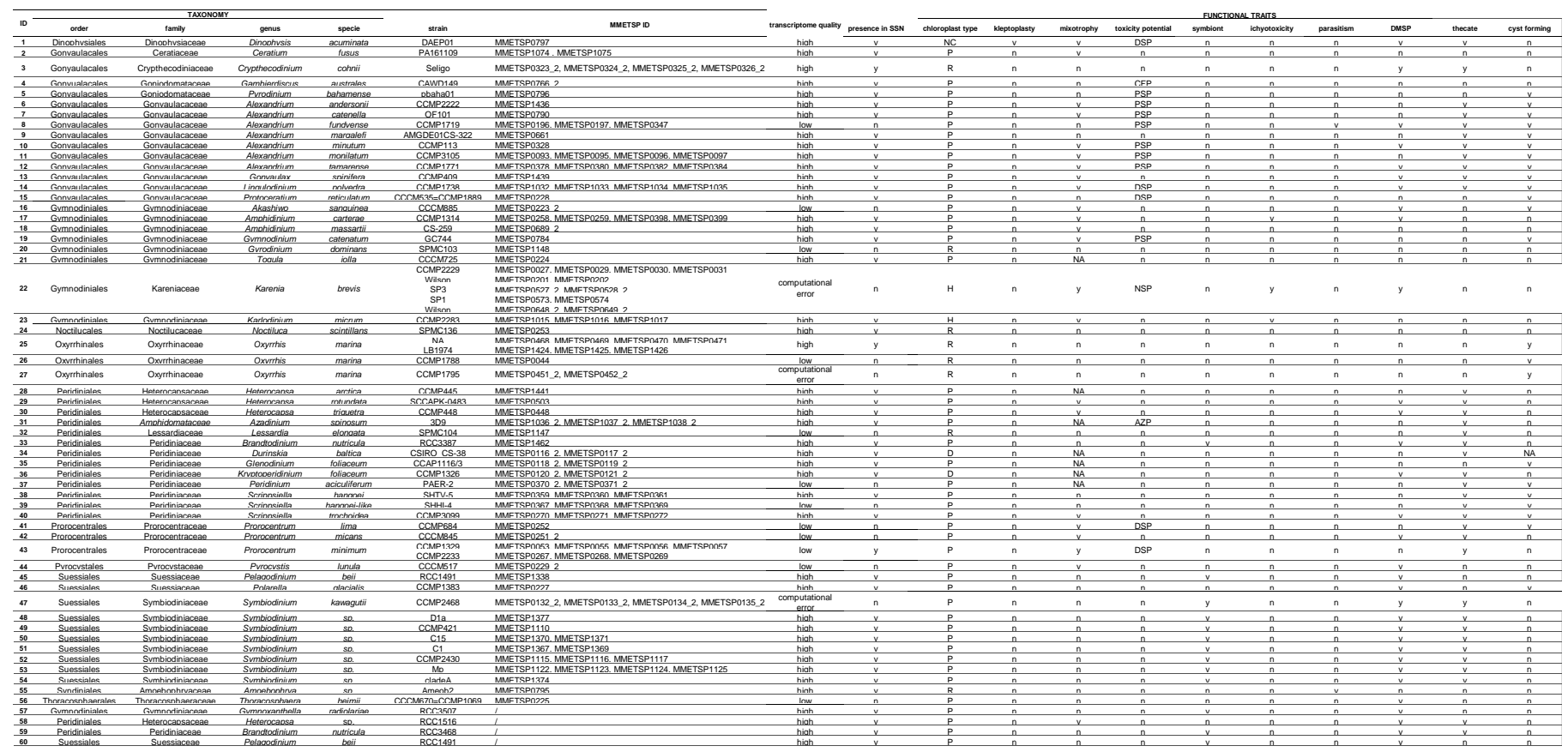




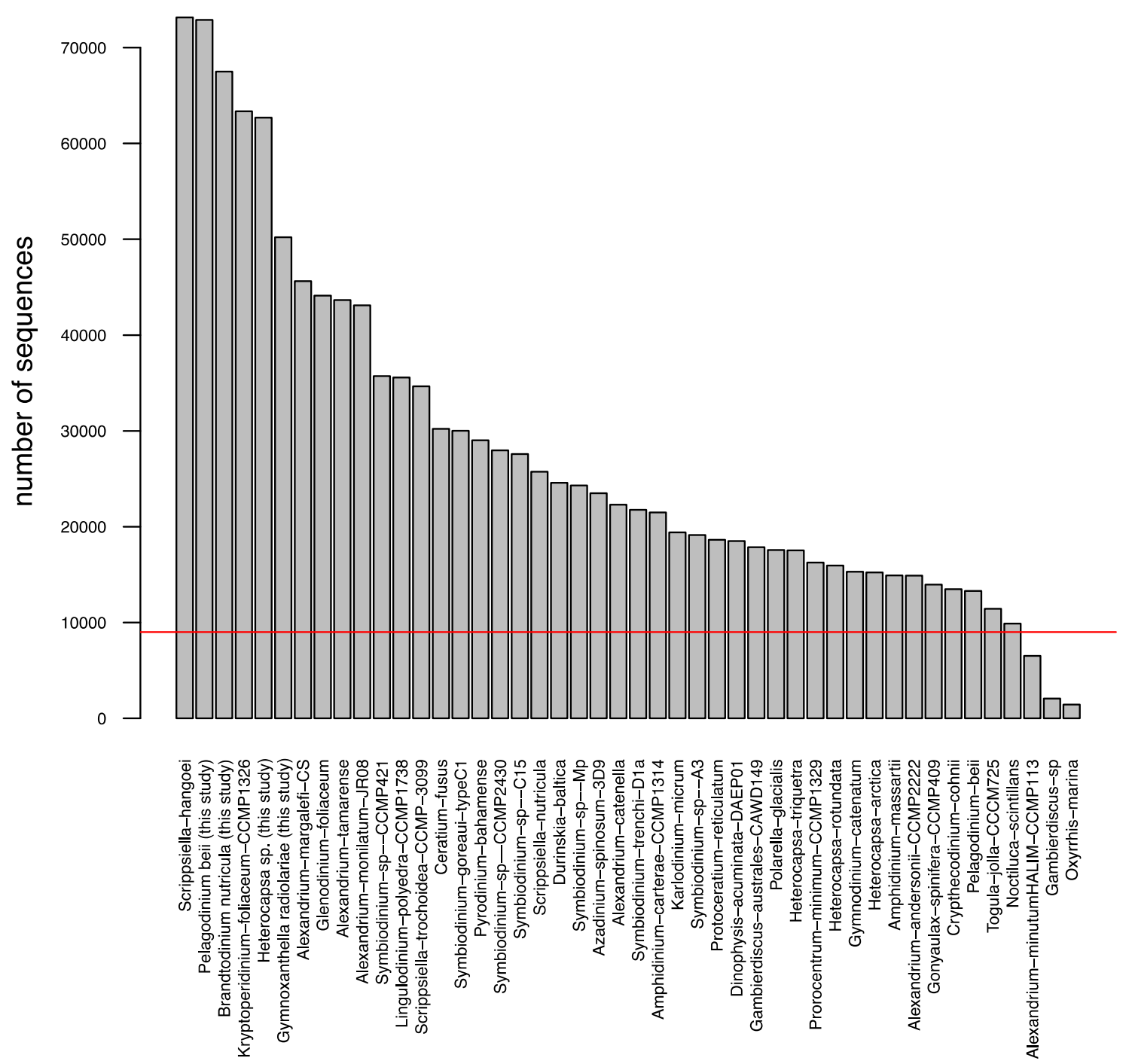

Fig. S 8: Number of peptide sequences per proteome derived from "high quality" transcriptomes. Red line represents the minimum number of sequences threshold (9,000 peptide sequences) required to perform core/accessory/pan proteome analysis. 
kleptoplastidy

SI Appendix Tab. S5 : kleptoplatic trait-CCs

\begin{tabular}{|l|l|}
\hline Number of CC composed of kleptoplastic species sequences & Unannotated \\
\hline
\end{tabular}

\begin{tabular}{l|l|}
6995 & 4493 \\
\hline
\end{tabular}

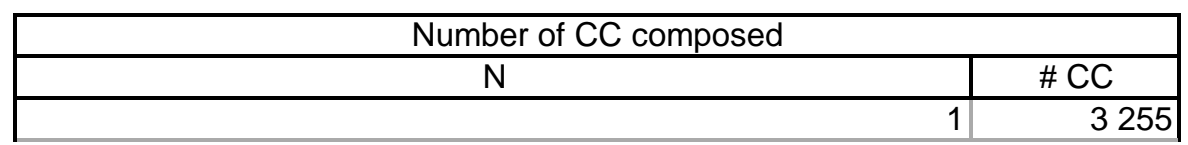

